\title{
Collective cell sorting requires contractile cortical waves in
}

\section{germline cells}

3

4

5

6

7 Soline Chanet ${ }^{1}$ and Jean-René Huynh ${ }^{1 *}$

8

91 Collège de France, PSL Research University, CNRS, Inserm, Center for

10 Interdisciplinary Research in Biology, Paris, France

11

12

*Corresponding author: jean-rene.huynh@ college-de-france.fr

13

14

15 Keywords: morphogenesis, adhesion, actin, myosin, oogenesis, Drosophila 


\section{ABSTRACT}

17 Encapsulation of germline cells by layers of somatic cells forms the basic unit of female

18 reproduction called primordial follicles in mammals and egg chambers in Drosophila. How

19 germline and somatic tissues are coordinated for the morphogenesis of each separated unit

20 remains poorly understood. Here, using improved live-imaging of Drosophila ovaries, we

21 uncovered periodic actomyosin waves at the cortex of germ cells. These contractile waves are

22 associated with pressure release blebs, which project from germ cells into somatic cells. We

23 demonstrate that these cortical activities, together with cadherin-based adhesion, are required

24 to sort each germline cyst as one collective unit. Genetic perturbations of cortical

25 contractility, blebs protrusion or adhesion between germline and somatic cells induced

26 failures to encapsulate any germ cells or the inclusion of too many germ cells or even the

27 mechanical split of germline cysts. Our results reveal that germ cells play an active role in the

28 physical coupling with somatic cells to produce the female gamete. 


\section{INTRODUCTION}

During development, tissues from different origins often cooperate and coordinate their morphogenetic movements to generate complex organs. Formation of the female gamete for example requires tight coordination between germ cells and the surrounding somatic tissue. During their differentiation, germ cells undergo several rounds of mitosis before entering meiosis. In most species, these mitoses are incomplete giving rise to cysts of cells interconnected by cytoplasmic bridges (Pepling et al., 1999). Each germline cyst is then surrounded by cells of somatic origin called pre-granulosa cells in mammals and follicle cells in Drosophila (Elkouby and Mullins, 2017). In mammals, pre-granulosa cells invade in between germ cells and each cyst eventually breaks down (CBD) into single cells encased by granulosa cells, forming primordial follicles (PFs) (Lei and Spradling, 2013). In Drosophila, egg chambers are made of precisely 16 germ cells surrounded by an epithelium of follicle cells (De Cuevas et al., 1997). Follicle cells do not invade the germline and intercellular bridges are maintained throughout oogenesis. Encapsulation is a conserved and important process to investigate as PFs and egg chambers give rise to the future female gamete, and defects in this step lead to sterility. Studies on the role of somatic cells during this step revealed general principles of epithelium morphogenesis (Godt and Tepass, 2003; HorneBadovinac and Bilder, 2005; Sarpal et al., 2012; St Johnston and Sanson, 2011). In contrast, the contribution of germ cells to encapsulation remains poorly characterized, and germ cells are assumed to be passive and transported by somatic cells.

In Drosophila, these early steps of oogenesis take place in a specialized structure called the germarium at the anterior tip of the ovary (Figure 1a) (Huynh and St Johnston, 2004). The germarium contains both germline and somatic stem cells, which divide to produce egg chambers throughout adult life. Germline stem cells (GSCs) are located at the most anterior tip of the region 1 of the germarium and give rise to cystoblasts. Cystoblasts then undergo four rounds of incomplete mitosis to generate cysts of 16 cells interconnected by ring canals. Only one cell per cyst becomes an oocyte and completes meiosis. The remaining 15 cells differentiate as nurse cells and synthesize nutrients and RNAs required for oocyte growth and maturation. Once made of 16 cells, germline cysts enter region $2 \mathrm{a}$, where they come into contact with follicle cells (FCs) of somatic origin produced by a population of follicle stem cells (FSCs) (Reilein et al., 2017). In region 2a, germline cysts are round, and several cysts can be found at similar stages of development. Then, only one cyst at a time moves to region $2 \mathrm{~b}$ and flattens to take the shape of a disc spanning the width of the 
germarium. All 16 cells of each cyst move collectively as a unit. It is during this transition from region $2 \mathrm{a}$ to $2 \mathrm{~b}$, that encapsulation starts. The 16 cells of each cyst become separated from other cysts by ingressing FCs that migrate centripetally (Horne-Badovinac and Bilder, 2005; Morris and Spradling, 2011). Moving posteriorly to region 3 (aka stage 1), cysts increase in volume and become round again, encased by a monolayer of around 30 epithelial FCs. FCs at both poles of the cyst intercalate and form stalks of cells, which pinch off the newly formed egg chamber from the germarium into the vitellarium (Morris and Spradling, 2011). In the vitellarium, egg chambers grow rapidly as separate units and polarize to become competent for fertilization.

How the morphogenetic movements of encapsulation are coordinated between germ cells and somatic cells is not known. Mechanical forces that shape cells and tissue are usually produced by actomyosin contractility where the molecular motor myosin 2 contract cortical filamentous actin (F-actin). To be effective, these contractile forces must be transmitted to the extracellular substrate or neighboring cells through links between F-actin network and junctional complexes, such as cadherins and integrins (Lecuit et al., 2011; Salbreux et al., 2012). In both mammals and flies, cadherins rather than integrins mediate interactions between somatic and germ cells at these early stages (Bendel-Stenzel et al., 2000; Godt and Tepass, 2003). In particular, in flies, E-Cadherin forms a gradient in both FCs and germ cells to help position the oocyte at the posterior of the egg chamber (Becam et al., 2005; Godt and Tepass, 1998; Gonzalez-Reyes and St Johnston, 1998). $\beta$-catenin (armadillo, arm in Drosophila) and $\alpha$-catenin link E-Cadherin to the underlying actomyosin cortex (Peifer et al., 1993; Sarpal et al., 2012; White et al., 1998).

In this study, we used hydrogel-based live imaging, genetics and image analyses to investigate the role of germ cells during the very first steps of encapsulation. We show that germline cysts actively generate forces both to maintain their position in the germarium while being surrounded by FCs, and to preserve their integrity as groups of 16 cells.

\section{RESULTS}

\section{1) Germ cells generate actomyosin traveling waves}

To investigate a potential role of germ cells during encapsulation, we first looked at actomyosin dynamics in the germline. To monitor the dynamics of the actomyosin cytoskeleton, we used a GFP-tagged version of the regulatory light chain of the non-muscle 
myosin 2 (sqh::GFP, and hereafter referred as myosin::GFP) expressed under its own promoter (Royou et al., 2002), and the actin reporters, LifeAct (that we expressed exclusively in the germline) or Utrophin (Utr), expressed ubiquitously (Huelsmann et al., 2013; Rauzi et al., 2010). Strikingly, we observed waves of myosin and F-actin at the cortex of germline cysts (Figure 1b-e, Movies 1-2). The frequency of these waves was not uniform across the germarium (Figure 1b, f). Wave frequency was high in GSCs with a low myosin intensity signal, and then faded away in the mitotic region. It became high again, and both more periodic and intense in region $2 \mathrm{a} / 2 \mathrm{~b}$. Interestingly, this increase in region $2 \mathrm{a} / 2 \mathrm{~b}$ corresponds to the first contacts between somatic follicle cells and germline cysts (Figure 1b, f). These results indicated that the cortical dynamics of the germ cells vary according to cyst differentiation. In region $2 b$ and region 3, we measured a period of the waves of 13,5 +/-1.2 min and $9.4+/-0.5$ min respectively. We found that $s q h:: D e n d r a 2$ photoconverted on one side of a cell could travel to the opposite side demonstrating that myosin was travelling (Figure S1a) (Roubinet et al., 2017). We measured an average speed of $0.028+/-0.0022 \mu \mathrm{m} . \mathrm{s}^{-1}$.

We next asked what regulates actomyosin waves. Cortical dynamics can be regulated by myosin activity, localization, F-actin network conformation (branched vs. linear) as well as polymerization and turn-over rates. Myosin activation often occurs downstream of RhoA and its effector, the myosin-activating kinase, Rho-associated coiled-coil kinase ROCK (rok in Drosophila) (Jaffe and Hall, 2005; Winter et al., 2001). We observed that ROCK also travelled as waves at the cortex of germ cells, but slightly ahead of myosin waves (Figure 1g, the time shift between ROCK and myosin waves was about 30s). To test if the activity of ROCK was required for the propagation of these waves, we made use of the chemical inhibitor Y27632. We used a water-based environment to be able to add this drug while recording. We adapted a live-imaging set up based on a photo-crosslinked PEG hydrogel, which could gently immobilize and maintain germarium morphology, while allowing diffusion of aqueous medium and drug treatments (Figure S1b) (Burnett et al., 2018). The addition of Y27632 stopped waves propagation within 1 to 2 min, indicating that ROCK activity was required for waves propagation (Figure 1h, i, Movie 3). As a control experiment, addition of water alone had no effect (Figure 1i).

Next, we tested the requirement for F-actin dynamics. We found that adding Cytochalasin D (Cyto-D), an inhibitor of actin turn-over, or CK-666, which inhibits Arp2/3 and actin branching, completely repressed waves propagation. In contrast, adding DMSO alone had no effect (Figure 1h, i, Movie 3). Finally, we found that addition of Colcemid, an 
131 inhibitor of microtubules polymerization, had no detectable effect on actomyosin waves 132 dynamics (Movie 4).

133 Collectively, these results describe periodic oscillations of the actomyosin cytoskeleton at

134 the cortex of germline cells; and demonstrate that waves propagation requires myosin activity 135 and actin polymerization.

2) High cortical contractility in germ cells is associated with pressure release blebs.

Strong contractions of the actomyosin network can induce ruptures of the cortex or its detachment from the plasma membrane (Charras and Paluch, 2008; Diz-Muñoz et al., 2013).

141 These ruptures lead to the formation of cytoplasmic protrusions, called blebs, which release cytoplasmic hydrostatic pressure. Blebs are thus signs of strong cortical contractility. We

143 often observed that following a wave of myosin, a break in the actomyosin meshwork formed 144 and allowed the expansion of bleb protrusions deep into the somatic cell layers. Blebs expansion left only a ring of myosin at the neck (Figure $1 \mathrm{j}$, Movie 5). The actomyosin meshwork then reformed inside the protrusion driving bleb retraction. Inhibiting cortical contractility by adding Cyto-D immediately eliminated blebs (Movie 6). This showed that blebs formation in germ cells was dependent on actomyosin contractility. We found that blebs frequency followed the increase in wave occurrences from region $2 \mathrm{~b}$ to region 3 (Figure $1 \mathrm{k}$ ). Noticeably, no blebbing was detected in region 1 of the germarium when cortical contractility is weak.

We concluded that oscillations of actomyosin at the germ cells cortex were contractile and associated with blebs. This high contractility in region $2 \mathrm{~b} / 3$ suggested that germline cells could play an active role in the encapsulation process.

\section{3) Alterations of cortical contractility in germ cells lead to the packaging of} abnormal numbers of germ cells

In order to test the functional significance of contraction waves, we reduced cortical 160 contractility in the germline, either by knocking down chickadee (chic-RNAi), the Drosophila 161 homolog of profilin, required for actin polymerization; or zipper (zip-RNAi), which encodes

162 for myosin 2 heavy chain. We achieved spatial and temporal specificity using either the 163 nanos-Gal4 or bam-Gal4 driver, which are only expressed in germline cells. The bam-Gal4 164 driver is weaker than nanos-Gal4, but allows knocking down genes in region 2a to region 3 of 
165 the germarium without interfering with stem cells and the formation of germline cysts.

166 Depleting chic or zip in germ cells effectively reduced cortical myosin intensity and waves

167 frequency compared to a control knocked-down (ctl-RNAi) (Figure 2a, b, Movie 7). We

168 obtained similar reduction of cortical contractility in germline cysts mutant for ROCK (rok ${ }^{2}$ )

169 (Figure S2a). We also tested the consequences of increasing cortical contractility by inhibiting

$170 \mathrm{mbs}$, the myosin binding subunit of the myosin phosphatase, which dephosphorylates and

171 inhibits myosin. Depleting $m b s$ in the germline ( $m b s-R N A i)$, increased waves frequency

172 (Figure 2a, b, Movie 7).

173 The most striking phenotype induced by both reducing or increasing contraction waves in

174 the germline was an abnormal number of germ cells per egg chamber, detected during mid-

175 oogenesis (12 to $36 \mathrm{~h}$ after leaving the germarium) (Figure 2c, d). Instead of egg chambers

176 containing 16 germ cells with one oocyte, mutant egg chambers were made of a number of

177 germ cells ranging from 0 to 32 cells, with 0,1 or 2 oocytes (Figure 2c, d, oocyte marked by

178 Orb). The abnormal number of germ cells could come from defects in mitosis of single-cell

179 precursors (abnormal number of divisions or defective abscission of GSCs/cystoblasts)

180 (Hawkins et al., 1996; Mathieu et al., 2013); or defects in cell sorting, with the encapsulation

181 of germ cells from different cysts into the same egg chamber. To distinguish between these

182 two hypotheses, we performed a cell-lineage analysis using the FLP-out technique to label

183 germline cysts generated by a single-cell precursor. In our experiment, RNAi expressing cysts

184 were GFP+ and wild type cysts GFP-. We found that egg chambers containing 32 germ cells

185 were made of two different 16-cell cysts packaged together, and not a single 32-cell cyst

186 caused by abnormal divisions (Figure 2e). Similar results were obtained with germline clones

187 mutant for $s q h^{1}$ (Figure S2b). We also found that egg chambers with less than 16 germ cells

188 were associated with neighboring egg chambers containing the missing complement of GFP+

189 germ cells (Figure 2e, 10 GFP+ germ cells in one egg chamber are associated with the

190 missing 6 GFP+ germ cells in the neighboring egg chamber). Long stalks of FCs correlated

191 with pseudo-egg chamber empty of any germ cell (Figure 2c).

192 We concluded that altering cortical contractility induced defects in sorting germline cysts

193 into groups of 16 cells and resulted in the formation of unfertile egg chambers with abnormal

194 numbers of germ cells. It further suggested that the cause of these phenotypes could be

195 encapsulation defects at earlier stages of oogenesis. 
To test if abnormal egg chambers originated from encapsulation defects, we imaged live the early steps of encapsulation in hydrogel. We followed the displacement of individual cysts along the anterior-posterior (a-p) axis, at the time they started to be separated by FCs (in region $2 b$ and 3 of the germarium). We used cap cells at the anterior tip of the germarium as a fixed reference point and measured cyst displacement by tracking their movements over 1 to 2h (Figure 3a, b, initial positions indicated by dotted lines and final positions with continuous line). In $c t l-R N A i$ conditions, we observed that when somatic cells ingressed to separate two cysts, the older cyst was slightly displaced toward the posterior of the germarium (positive orientation), and the younger cyst was slightly displaced toward the anterior (negative orientation) (Figure 3b, Figure S3, Movie 8). Overall cyst displacement was small, we measured a mean displacement speed of $0.0125+/-0.0019 \mu \mathrm{m} . \mathrm{min}^{-1}$, which is in accordance with previous recording of wild-type cyst movements (Morris and Spradling, 2011).

In chic-RNAi and zip-RNAi conditions, however, cysts movements were significantly increased and we measured a mean displacement speed of $0.0285+/-0.0022 \mu \mathrm{m} . \mathrm{min}^{-1}$ and $0.0237+/-0.0024 \mu \mathrm{m} \cdot \mathrm{min}^{-1}$ respectively (Figure 3b, Figure S3). Cysts in regions $2 \mathrm{~b}$ and 3 were frequently pushed back together more anteriorly (Figure 3a, zip-RNAi upper panel). These increased movements resulted in collisions between cysts in the germarium and formation of long stretches of FCs. If collisions were not resolved at the time of egg chamber individualization, this would lead to the formation of a compound egg chamber containing two cysts packaged together. Thus, cortical contractility is required to prevent uncontrolled germline cysts movement and collision between cyst at the time of encapsulation.

In chic-RNAi and zip-RNAi, we also noticed that cysts in region 3 instead of being round adopted an elongated shape along the a-p axis compared to controls (Figure 3c, increased aspect ratio). Live-imaging showed cysts being squeezed and sometimes cut into several parts by ingressing FCs (Figure 3a, zip-RNAi lower panel, Movie 8 and 9). Cysts splitting events would give rise to egg chambers with less than 16 germ cells. In addition, different parts of the split cysts could also be packaged with adjacent cysts generating egg chambers with more than 16 cells. These results indicated that cortical contractility also conferred stiffness to germline cysts, preventing them from being squeezed and cut by surrounding FCs.

In $m b s$-RNAi, cysts movements and speed were also increased compared to control conditions. We measured a mean displacement speed of $0.0203+/-0.0029 \mu \mathrm{m} \cdot \mathrm{min}^{-1}$. Whereas in zip-RNAi and chic-RNAi cysts tend to be more frequently pushed toward the anterior, $m b s$ RNAi cysts tend to move most frequently toward the posterior (Figure $3 \mathrm{~b}$ ). This behavior was more pronounced for cysts in region 3 , that significantly moved faster toward the posterior 
than $c t l-R N A i$ in region 3 (Figure S3). In the strongest instances, we observed collisions in the posterior regions of the germarium between a fast moving cyst and an older cyst resulting in the encapsulation of the two cysts together (Movie 9). In contrast to chic-RNAi and zip-RNAi, we never observed cysts being split in $m b s-R N A i$. $m b s$-RNAi cysts were not squeezed but remained round in region 3 with an aspect ratio close to 1 (Figure $3 \mathrm{c}$ ). These results suggest that increasing cortical contractility in $m b s-R N A i$ favor faster displacement of the cysts toward the posterior of the germarium.

Together, our live-imaging experiments showed that cortical contractility is required for correct positioning of cysts at the time of encapsulation both to avoid collisions between cysts and formation of long stalks of FCs devoid of germ cells. Germline contractility is also required to maintain cyst integrity as a group of 16 cells and avoid cyst splitting. These observations helped explain our cell-lineage analysis. Thus, we concluded that alteration of cortical contractility in germ cells induced a loss of coordination between germline and somatic cells movements, leading to encapsulation of abnormal numbers of germ cells. Next, we looked for links between germ cells and somatic cells that could mediate this coordination.

\section{5) Cadherin-based adhesion is required for correct encapsulation}

During oogenesis, interactions between germ cells and somatic cells are mediated by ECadherin (E-Cad) homophilic interactions (Godt and Tepass, 1998; Gonzalez-Reyes and St Johnston, 1998). We also showed previously that adherens junctions are present between germ cells around ring canals (Figure 4a, Movie 10) (Fichelson et al., 2010). $\beta$-catenin (armadillo, arm in Drosophila) and $\alpha$-catenin link E-Cadherin to the underlying actomyosin cortex (Peifer et al., 1993; Sarpal et al., 2012; White et al., 1998).

To decrease homophilic interactions between germ and somatic cells, we depleted ECad (shotgun, shg in Drosophila) either in the germline using the bam-Gal 4 driver (ECadRNAi germ, Figure S3a) or in the FCs using traffic jam-Gal4, which is strongly expressed in FCs (ECad-RNAi soma, Figure S3a). We used E-Cad-shRNA to avoid functional compensation by N-Cad (see Material and Methods and (Loyer et al., 2015)). We found that it induced encapsulation phenotypes, similar to those observed after knocked-down of zip or chic in the germline. On fixed ovaries, we found egg chambers made of two 16-cell cysts, or separated by empty stalk cells (Figure 4d, e). In addition, when E-Cad was depleted in germ cells, we found a majority of egg chambers with fewer germ cells, indicating that groups of 16 cells had been split between several egg chambers (Figure 4d). As in chic-RNAi or zip-RNAi, 
occurrence of split cysts correlated with strong deformations of ECad-RNAi germ mutant cysts along the a-p axis in region 3 (Figure 4f). However, egg chambers containing split cyst were not observed when E-Cad was depleted in FCs only, and cyst aspect ratio in region 3 was not affected in this case (Figure 4f).

Live imaging confirmed these encapsulation defects: we observed increased cyst movements in region $2 \mathrm{~b}$ and 3 of the germarium (mean displacement speed $=0.0427+/$ $0.0044 \mu \mathrm{m} . \mathrm{min}^{-1}$ for ECad-RNAi germ and $0.0507+/-0.0066 \mu \mathrm{m} . \mathrm{min}^{-1}$ for ECad-RNAi soma), with cysts being pushed toward the anterior or the posterior leading to collisions with the preceding or following cyst (Figure 4g, h, Figure S4b, Movie 11). We also observed cysts being deformed along the a-p axis and split by FCs when E-Cad was knock-downed in the germline (Movies 12). Similar phenotypes were observed although with a lower penetrance after knock-down of arm (arm-RNAi) in the germline (Figure S4c, Movie 12). Consistent with our results, encapsulation defects were also reported with mutant alleles of $\beta$-catenin and $\alpha$ catenin (Peifer et al., 1993; Sarpal et al., 2012; White et al., 1998).

These results showed that reducing cell adhesion in either germ cells or somatic cells gave similar encapsulation phenotypes than impairing cortical contractility in germ cells. We thus tested whether reducing cell adhesion affected germline cells contractility. Quantification of actomyosin waves frequency showed that they were not affected in ECad-RNAi mutant cysts (Figure 4b, c, Movie 13). Eliminating cell adhesion thus does not noticeably impact germ cells contractility. Junctional complexes, however, are required to transmit contractile forces to neighboring cells (Martin et al., 2010).

We concluded that correct encapsulation requires generation of contractile forces by germline cysts and adhesion between germ cells to prevent cysts splitting. It also requires transmission of these contractile forces to follicle cells layer through E-Cad adhesion complexes to maintain cyst position and prevent collisions between cysts.

\section{6) Altering blebs frequency leads to encapsulation defects.}

Since blebs are direct consequences of strong cortical contractions and in direct contact with FCs, we investigated whether blebs were also involved in encapsulation and cyst positioning. To manipulate blebs occurrences without directly affecting actomyosin contractility, we thought of modifying properties of the cortex by expressing two different mutant forms of Moesin in germ cells. Moesin is the sole ERM (Ezrin Radixin Moesin) protein in Drosophila and links the actomyosin cortex to the plasma membrane. Its activity is 
regulated by phosphorylation at T559 (Kunda et al., 2008). When we expressed a nonphosphorylatable form (moe-TA::GFP) in germ cells, known for its dominant-negative function, we noticed an increase in the average number of blebs per cyst (Figure 5a, b). This correlated with encapsulation defects and the formation of egg chambers with abnormal numbers of germ cells (Figure 5c, d). Cell lineage analysis further showed that GFP+ cysts expressing moe-TA::GFP could be found packaged with a wild-type cyst in a compound egg chamber (Figure 5c). We then looked by live-imaging how these defects emerged. Interestingly, we observed that moe-TA::GFP overexpressing cysts in region 3 moved more and faster (with a mean displacement speed of $0.0187+/-0.0028$ ) toward the posterior than control cysts (mean displacement speed $=0.0107+/-0.0028$ ) (Figure 5e). A highly blebbing cyst could sometimes contact and invade a posterior cyst leading to cysts collision (Figure 5f, Movies 14, 15).

On the other hand, expressing a phosphomimetic form of Moesin (moe-TD::GFP) in germ cells is thought to induce a stiffer cortex. We observed smaller blebs and a decrease in number of blebs, although not statistically significant (Figure 5a, b). In contrast to cysts expressing moe-TA::GFP, cysts expressing moe-TD::GFP were not able to significantly move forward and only induced mild encapsulation defects (Figure 5d, e).

These results showed that blebs could play a role during encapsulation. Increasing bleb occurrences was sufficient to accelerate germline cysts movement, which can induce collisions and encapsulation defects. Thus, our results suggest that increasing blebs in germline cysts could induce a bleb-based motility behavior.

7) Germline cysts play an active role in cysts sorting using migration-like mechanisms.

To reveal a putative germ cells autonomous role in cyst positioning during encapsulation, we aimed to block somatic cells movement and thus suppress constriction forces exerted on germline cysts. We did so by mechanically blocking FCs centripetal migration. We observed that when we mounted germarium in halocarbon oil (10S), FCs strongly adhere to the coverslip and were unable to migrate. In normal conditions, FCs convergent-extensions movements constrict the germarium in-between cysts progressively reducing the width of the stalk that will separate the future egg chambers (Figure 6a) (Morris and Spradling, 2011). In

333 hydrogel we measured a reduction of $4.5+/-0.5 \%$ of the stalk width in $50 \mathrm{~min}$. In oil, FCs 334 tend instead to slightly expand on the coverslip resulting in an expansion of $0.6+/-1.0 \%$ of 
the stalk width (Figure 6a). FCs were thus not able to intercalate and constrict underlying germ cells. In these conditions, we measured positive movement of germline cysts toward the posterior of the germarium, indicating that wild-type germline cysts were able to migrate on stalled FCs, which can lead to collision between cysts (Figure 6b, c, Movie 16). Knock-down of zip or E-Cad in the germline however significantly reduced germline cyst movement and speed in germarium mounted in oil (Figure 6b). This indicated that the ability of cysts to migrate on stalled FCs depends on cortical contractility and adhesion with surrounding FCs. Importantly, these results also revealed that fast displacement of germline cysts with reduced cortical contractility or adhesion observed in hydrogel were passive and imposed by surrounding FCs constriction forces (Figure 3b and Figure 4g). Mutant cysts were passively pushed backward or forward as somatic cells rearranged and constricted to form a stalk (Figure S5).

Thus, our data suggest that cortical contractility in germ cells can generate forces enabling cysts to migrate. To support this idea, we compared two populations of germline cysts within the same germarium using clonal analysis. We induced mosaic germaria containing germline cysts mutant for rok and marked by the absence of RFP (wild-type cysts are RFP+) (Figure 6d). rok mutant cysts showed a clear reduction in waves frequency compared to wild type cysts (Figure S2a). In these conditions, wild type cysts invaded into posterior rok mutant cysts, indicating that wild type cysts were consistently faster to the posterior than rok mutant cysts (Figure 6d, Movie 17). Consistently, we never detected the reverse invasion of wild type cysts by mutant cysts ( $\mathrm{n}=6$ invasions of $r o k^{2}$ mutant cysts by wild-type cysts, 9 mosaic germaria).

Altogether, these results suggest that in normal conditions, germline cysts use migrationlike mechanisms to maintain their position within the germarium during encapsulation. Their autonomous movement toward the posterior is usually masked by the prominent movements and constriction of FCs around them that tend to push neighboring cysts in opposite direction to separate them. However, if we mechanically block FCs movements, germline cysts can visibly migrate forward (Figure 7).

\section{$\underline{\text { DISCUSSION }}$}

Our study revealed the existence of periodic contractile waves of the actomyosin network at the cortex of germ cells, as described in other developmental systems (Bement et al., 2015; Maitre et al., 2015; Weiner et al., 2007). The nature of these waves is dual, 
requiring both motor activity and actin polymerization. We demonstrated that these contractions are required to maintain correct positioning of germline cysts during encapsulation by FCs and to maintain their integrity as groups of 16 cells. In light of our results, we propose that there are at least two kinds of forces at play during encapsulation (Figure 7). Convergent-intercalation of somatic cells exerts constriction forces on the underlying germline cells, while contractility and protrusions of germ cells exert propelling forces on the overlying FCs. Correct encapsulation requires a proper balance between these forces. Indeed, on the one hand, when germ cells contractility was weakened, somatic cells could squeeze and cut or displace germline cysts. On the other hand, when somatic cells convergence was blocked, germline cells migrated faster and collided. We further showed that E-Cadherin-based adhesion between germline and somatic cells is required to transmit forces and to coordinate both morphogenetic movements.

Mechanistically, we propose that contractile waves contribute to the dynamics of cellular adhesion between germline and somatic cells. Indeed, knocking down contractility in germ cells or cadherin-based adhesion between germline cysts and FCs both led to increased and uncontrolled movements of germline cysts. This resulted in encapsulation defects with the packaging of several cysts in the same egg chamber, or long stalk of FCs without germ cells. We propose that cortical contractility at the interface between germline cyst and FCs helps to remodel dynamically cellular adhesion and thus to coordinate germline and somatic cells morphogenetic movements. This could involve junctional strengthening under tension or contractility-dependent E-Cad turnover and junction remodeling (Cavey et al., 2008; Liu et al., 2010; Shigenobu et al., 2010). In addition, friction forces could be generated between germline and somatic cells by flows of actomyosin linked to transmembrane E-cad proteins. This mechanism would be reminiscent of single cell migration mechanisms used in confined or crowded environments (Bergert et al., 2015; Hawkins et al., 2011; Paluch et al., 2016).

Reducing contractility or adhesion specifically in germ cells induced an additional phenotype whereby germline cysts were deformed along the anterior-posterior axis and split by ingressing somatic cells. This phenotype was not seen (or very rarely) when removing cadherin in somatic cells only. It indicates that adhesion and contractility in germ cells are required to sort cysts into group of 16 cells and to resist constriction forces applied by somatic cells. Adhesion and contractility are commonly assumed to function in cell sorting by controlling tissue surface tension (Krens and Heisenberg, 2011; Maitre et al., 2012; Manning et al., 2010). We propose that a similar mechanism operates here, where contractility and adhesion between cells within a cyst favor the formation of a stiff sphere that cannot be split 
during encapsulation. Interestingly, the abnormal packaging of germ cells in flies with reduced adhesion or contractility in the germline, is similar to the normal aggregation of germ cells from different cysts into nests that occurs in mice oogenesis (Lei and Spradling, 2013). Our results suggest that simple differences in the regulation of cellular adhesion or cortical contractility could explain this evolutionary difference between mice and flies.

We also showed that germline cysts are blebbing during encapsulation. The importance of blebs for cell migration has been clearly demonstrated in several cases (Liu et al., 2015; Paluch and Raz, 2013; Paluch et al., 2006; Ruprecht et al., 2015). The underlying mechanisms and physical models, however, vary. One model postulates that blebs could engage in cell-cell adhesion with its environment and allow the forward transfer of cytoplasm. It has also been proposed that in a confined environment, blebs could push laterally allowing cells "to chimney" their way forward. Our results are compatible with a role of blebs in generating propelling forces during encapsulation. We found that increasing blebs frequency can induce posterior cyst migration. The underlying physical model remains, however, to be investigated. Together, our results suggest that germline cysts use migration-like mechanism such as blebbing and DE-Cad based friction with the somatic layer to maintain their position in the germarium during encapsulation, counterbalancing the forces exerted by ingressing FCs.

The reverse situation of somatic follicle cells migrating in between germ cells has been well characterized in later stages of Drosophila oogenesis. A small group of anterior follicle cells, called border cells (BCs), detach from the anterior pole of the egg chamber and migrate between nurse cells to reach the oocyte (Montell, 2003). Like encapsulation, this process is collective and requires DE-Cadherin, actin polymerization and myosin activity. However, despite these similarities, BCs follow a mesenchymal-like mode of migration by extending long protrusions toward the oocyte (Mishra et al., 2019). In contrast, we found that germ cells contractility induces the formation of blebs, which are associated with amoeboid migration (Lammermann and Sixt, 2009). Our results are more reminiscent of primordial germ cells migration described in zebrafish, indicating that blebs formation may be a conserved property of germline cells (Blaser et al., 2006).

Our results are a first step toward a mechanical understanding of encapsulation. Mechanical signals can instruct and pattern cell behaviors (Chanet and Martin, 2014). In light of our study, we can speculate that mechanical feedbacks between germ cells and somatic cells could play a role in self-organizing encapsulation. An important issue is to address how these mechanical inputs are integrated and regulated by biochemical signaling pathways. In 
437 both mice and flies, there is a wealth of literature describing cell-cell communications

438 between somatic cells, and between germline and somatic cells at these stages. In Drosophila,

439 disruptions of the Hedgehog, Wingless, Notch, Jak/Stat or EGF pathways, all lead to

440 encapsulation defects with the formation of compound egg chambers with multiple cysts or

441 long stalks devoid of germ cells (Bastock and St Johnston, 2008; Klusza and Deng, 2011;

442 Roth and Lynch, 2009). These phenotypes have been attributed mostly to defects in cell fate

443 specifications. In light of our study, these signaling pathways could also have a more direct

444 role and regulate actomyosin contractility or adhesion, as their disruption induce similar

445 defects. It will be exciting to re-analyze these biochemical and mechanical activities as well

446 as their interplay during encapsulation. An integrated model of encapsulation will clearly

447 benefit our understanding of gamete formation and reproductive biology.

\section{Acknowledgments:}

450

451 We are grateful to Juliette Mathieu for the initial observations of germline cysts being split in 452 shg RNAi. We thank A. Guichet, A.C. Martin, A. Royou, the Bloomington Drosophila Stock 453 Center and the Developmental Studies Hybridoma Bank for kindly providing flies and 454 antibodies used in this study. We thank all members of the J-R. H. lab, F. Schweisguth, M-E. 455 Terret and M. Malartre for discussions and helpful comments on the manuscript. S.C. is

456 supported by an ARC postdoc fellowship and work in JRH lab is supported by CNRS, 457 Inserm, Collège de France, FRM (Equipe FRM DEQ20160334884), ANR (ANR-15-CE13458 0001-01, AbsCyStem) and Bettencourt-Schueller foundations. 


\section{REFERENCES}

Bardet, P.-L., G. Boris, P. Camille, S. Fanny, L. Valentine, B. Floris, G. Yûki, M. Vincent, G. François, and Y. Bellaïche. 2013. PTEN Controls Junction Lengthening and Stability during Cell Rearrangement in Epithelial Tissue. Developmental Cell. 25:534-546.

Bastock, R., and D. St Johnston. 2008. Drosophila oogenesis. Curr Biol. 18:R1082-1087.

Becam, I.E., G. Tanentzapf, J.A. Lepesant, N.H. Brown, and J.R. Huynh. 2005. Integrinindependent repression of cadherin transcription by talin during axis formation in Drosophila. Nature Cell Biol. 7:510-516.

Bement, W., M., L. Marcin, M.M. Alison, M.K. Angela, E.L. Matthew, E.G. Adriana, P. Courtney, S. Kuan-Chung, L.M. Ann, B.G. Andrew, and G. von Dassow. 2015. Activator-inhibitor coupling between Rho signalling and actin assembly makes the cell cortex an excitable medium. Nature Cell Biology. 17:1471-1483.

Bendel-Stenzel, M.R., M. Gomperts, R. Anderson, J. Heasman, and C. Wylie. 2000. The role of cadherins during primordial germ cell migration and early gonad formation in the mouse. Mech Dev. 91:143-152.

Bergert, M., A. Erzberger, R.A. Desai, I.M. Aspalter, A.C. Oates, G. Charras, G. Salbreux, and E.K. Paluch. 2015. Force transmission during adhesion-independent migration. Nat Cell Biol. 17:524-529.

Blaser, H., M. Reichman-Fried, I. Castanon, K. Dumstrei, F.L. Marlow, K. Kawakami, L. Solnica-Krezel, C.P. Heisenberg, and E. Raz. 2006. Migration of zebrafish primordial germ cells: a role for myosin contraction and cytoplasmic flow. Dev Cell. 11:613-627.

Burnett, K., E. Edsinger, and D.R. Albrecht. 2018. Rapid and gentle hydrogel encapsulation of living organisms enables long-term microscopy over multiple hours. Communications Biology. 1.

Cavey, M., M. Rauzi, P.-F. Lenne, and T. Lecuit. 2008. A two-tiered mechanism for stabilization and immobilization of E-cadherin. Nature. 453:751.

Chanet, S., and A.C. Martin. 2014. Mechanical force sensing in tissues. Prog Mol Biol Transl Sci. 126:317-352.

Charras, G., and E. Paluch. 2008. Blebs lead the way: how to migrate without lamellipodia. Nat Rev Mol Cell Biol. 9:730-736.

Clemot, M., A. Molla-Herman, J. Mathieu, J.R. Huynh, and N. Dostatni. 2018. The replicative histone chaperone CAF1 is essential for the maintenance of identity and genome integrity in adult stem cells. Development. 145.

De Cuevas, M., M. Lilly, and A. Spradling. 1997. Germline cysts formation in Drosophila. Annual review in Genetics.

Diz-Muñoz, A., D. Fletcher, A., and O. Weiner, D. 2013. Use the force: membrane tension as an organizer of cell shape and motility. Trends in Cell Biology. 23:47-53.

Elkouby, Y.M., and M.C. Mullins. 2017. Coordination of cellular differentiation, polarity, mitosis and meiosis - New findings from early vertebrate oogenesis. Dev Biol. 430:275-287.

Godt, D., and U. Tepass. 1998. Drosophila oocyte localization ismediated by differential cadherin-basedadhesion. Nature. 395:387-391.

Godt, D., and U. Tepass. 2003. Organogenesis: keeping in touch with the germ cells. Current Biology. 13:R683-R685.

Gonzalez-Reyes, A., and D. St Johnston. 1998. The Drosophil aAP axis is polarised by the cadherin-mediated positioning of the oocyte. Development. 125:3635-3644.

Hawkins, N.C., J. Thorpe, and T. Schupbach. 1996. encore, a gene required for the regulation of germ line mitosis and oocyte differentiation during Drosophila oogenesis. Development. 122:281-290. 
Hawkins, R.J., R. Poincloux, O. Benichou, M. Piel, P. Chavrier, and R. Voituriez. 2011. Spontaneous contractility-mediated cortical flow generates cell migration in threedimensional environments. Biophys J. 101:1041-1045.

Horne-Badovinac, S., and D. Bilder. 2005. Mass transit: Epithelial morphogenesis in the Drosophila egg chamber. Dev Dynam. 232:559-574.

Huang, J., Z. Wenke, D. Wei, M.W. Annie, and Y. Hong. 2009. Directed, efficient, and versatile modifications of the Drosophila genome by genomic engineering. Proc National Acad Sci. 106:8284-8289.

Huelsmann, S., J. Ylanne, and N.H. Brown. 2013. Filopodia-like actin cables position nuclei in association with perinuclear actin in Drosophila nurse cells. Dev Cell. 26:604-615.

Huynh, J.R., and D. St Johnston. 2004. The origin of asymmetry: early polarisation of the Drosophila germline cyst and oocyte. Curr Biol. 14:R438-449.

Jaffe, A., B., and A. Hall. 2005. RHO GTPASES: Biochemistry and Biology. Cell Dev Biology. 21:247-269.

Karess, R.E., X.J. Chang, K.A. Edwards, S. Kulkarni, I. Aguilera, and D.P. Kiehart. 1991. The regulatory light chain of nonmuscle myosin is encoded by spaghetti-squash, a gene required for cytokinesis in Drosophila. Cell. 65:1177-1189.

Klusza, S., and W.M. Deng. 2011. At the crossroads of differentiation and proliferation: precise control of cell-cycle changes by multiple signaling pathways in Drosophila follicle cells. Bioessays. 33:124-134.

Krens, S.F., and C.P. Heisenberg. 2011. Cell sorting in development. Curr Top Dev Biol. 95:189-213.

Kunda, P., A. Pelling, E., L. Tao, and B. Baum. 2008. Moesin Controls Cortical Rigidity, Cell Rounding, and Spindle Morphogenesis during Mitosis. Current Biology. 18:91-101.

Lammermann, T., and M. Sixt. 2009. Mechanical modes of 'amoeboid' cell migration. Curr Opin Cell Biol. 21:636-644.

Lecuit, T., P.-F. Lenne, and E. Munro. 2011. Force Generation, Transmission, and Integration during Cell and Tissue Morphogenesis. Annu Rev Cell Dev Bi. 27:157-184.

Lei, L., and A.C. Spradling. 2013. Mouse primordial germ cells produce cysts that partially fragment prior to meiosis. Development. 140:2075-2081.

Liu, Y.-J., M. Le Berre, L. Franziska, M. Paolo, C.-J. Andrew, H. Mélina, T. Tohru, R. Voituriez, and M. Piel. 2015. Confinement and Low Adhesion Induce Fast Amoeboid Migration of Slow Mesenchymal Cells. Cell. 160:659-672.

Liu, Z., L.T. John, M.C. Daniel, T.Y. Michael, J.S. Nathan, R. Sami Alom, M.N. Celeste, and C. Chen, S. 2010. Mechanical tugging force regulates the size of cell-cell junctions. Proc National Acad Sci. 107:9944-9949.

Loyer, N., I. Kolotuev, M. Pinot, and R. Le Borgne. 2015. Drosophila E-cadherin is required for the maintenance of ring canals anchoring to mechanically withstand tissue growth. Proc Natl Acad Sci U S A. 112:12717-12722.

Maître, J.-L., N. Ritsuya, H. Turlier, F. Nédélec, and T. Hiiragi. 2015. Pulsatile cellautonomous contractility drives compaction in the mouse embryo. Nature Cell Biology. 17:849-855.

Maitre, J.L., H. Berthoumieux, S.F. Krens, G. Salbreux, F. Julicher, E. Paluch, and C.P. Heisenberg. 2012. Adhesion functions in cell sorting by mechanically coupling the cortices of adhering cells. Science. 338:253-256.

Manning, M.L., R.A. Foty, M.S. Steinberg, and E.M. Schoetz. 2010. Coaction of intercellular adhesion and cortical tension specifies tissue surface tension. Proc Natl Acad Sci U S A. 107:12517-12522.

Martin, A., C., M. Kaschube, and E. Wieschaus, F. 2008. Pulsed contractions of an actinmyosin network drive apical constriction. Nature. 457:495. 
584

585

586

587

588

589

590

591

592

593

594

595

596

597

598

599

600

601

602

603

604

605

606

607

Martin, A.C., M. Gelbart, R. Fernandez-Gonzalez, M. Kaschube, and E.F. Wieschaus. 2010. Integration of contractile forces during tissue invagination. J Cell Biol. 188:735-749.

Mathieu, J., C. Cauvin, C. Moch, S.J. Radford, P. Sampaio, C.N. Perdigoto, F. Schweisguth, A.J. Bardin, C.E. Sunkel, K. McKim, A. Echard, and J.R. Huynh. 2013. Aurora B and cyclin B have opposite effects on the timing of cytokinesis abscission in Drosophila germ cells and in vertebrate somatic cells. Dev Cell. 26:250-265.

Mishra, A.K., J.A. Mondo, J.P. Campanale, and D.J. Montell. 2019. Coordination of protrusion dynamics within and between collectively migrating border cells by myosin II. Mol Biol Cell. 30:2490-2502.

Montell, D.J. 2003. Border-cell migration: the race is on. Nat Rev Mol Cell Biol. 4:13-24.

Morin, X., D. Richard, Z. Michael, and W. Chia. 2001. A protein trap strategy to detect GFPtagged proteins expressed from their endogenous loci in Drosophila. Proc National Acad Sci. 98:15050-15055.

Morris, L.X., and A.C. Spradling. 2011. Long-term live imaging provides new insight into stem cell regulation and germline-soma coordination in the Drosophila ovary. Development. 138:2207-2215.

Paluch, E., K., and E. Raz. 2013. The role and regulation of blebs in cell migration. Curr Opin Cell Biol. 25:582-590.

Paluch, E., C. Sykes, J. Prost, and M. Bornens. 2006. Dynamic modes of the cortical actomyosin gel during cell locomotion and division. Trends Cell Biol. 16:5-10.

Paluch, E.K., I.M. Aspalter, and M. Sixt. 2016. Focal Adhesion-Independent Cell Migration. Annu Rev Cell Dev Biol. 32:469-490.

Peifer, M., S. Orsulic, D. Sweeton, and E. Wieschaus. 1993. A role for the Drosophila segment polarity gene armadillo in cell adhesion and cytoskeletal integrity during oogenesis. Development. 118:1191-1207.

Pepling, M.E., M. de Cuevas, and A. Spradling. 1999. Germline cysts: a conserved phase of germ cell development? Trends in Cell Biology. 9:259.

Rauzi, M., P.F. Lenne, and T. Lecuit. 2010. Planar polarized actomyosin contractile flows control epithelial junction remodelling. Nature. 468:1110-1114.

Reilein, A., D. Melamed, K.S. Park, A. Berg, E. Cimetta, N. Tandon, G. Vunjak-Novakovic, S. Finkelstein, and D. Kalderon. 2017. Alternative direct stem cell derivatives defined by stem cell location and graded Wnt signalling. Nat Cell Biol. 19:433-444.

Roth, S., and J.A. Lynch. 2009. Symmetry breaking during Drosophila oogenesis. Cold Spring Harb Perspect Biol. 1:a001891.

Roubinet, C., A. Tsankova, T.T. Pham, A. Monnard, E. Caussinus, M. Affolter, and C. Cabernard. 2017. Spatio-temporally separated cortical flows and spindle geometry establish physical asymmetry in fly neural stem cells. Nat Commun. 8:1383.

Royou, A., W. Sullivan, and R. Karess. 2002. Cortical recruitment of nonmuscle myosin II in early syncytial Drosophila embryos: its role in nuclear axial expansion and its regulation by Cdc2 activity. J Cell Biol. 158:127-137.

Ruprecht, V., W. Stefan, C.-J. Andrew, S. Michael, M. Hitoshi, S. Keisuke, B. Vanessa, R.M. Monika, S. Michael, R. Voituriez, and C.-P. Heisenberg. 2015. Cortical Contractility Triggers a Stochastic Switch to Fast Amoeboid Cell Motility. Cell. 160:673-685.

Salbreux, G., G. Charras, and E. Paluch. 2012. Actin cortex mechanics and cellular morphogenesis. Trends in Cell Biology. 22:536-545.

Sarpal, R., M. Pellikka, R.R. Patel, F.Y. Hui, D. Godt, and U. Tepass. 2012. Mutational analysis supports a core role for Drosophila alpha-catenin in adherens junction function. J Cell Sci. 125:233-245. 
Shigenobu, Y., W. Yuko, W. Toshiyuki, N. Akira, and S. Mai. 2010. $\alpha$-Catenin as a tension transducer that induces adherens junction development. Nature Cell Biology. 12:533542.

611 St Johnston, D., and B. Sanson. 2011. Epithelial polarity and morphogenesis. Curr Opin Cell Biol. 23:540-546.

613

614

615

Van Doren, M., A. Williamson, L., and R. Lehmann. 1998. Regulation of zygotic gene expression in Drosophila primordial germ cells. Current Biology. 8:243-246.

Weiner, O., D., A.M. William, F.W. Lani, J.A. Steven, and M. Kirschner, W. 2007. An ActinBased Wave Generator Organizes Cell Motility. Plos Biol. 5:e221.

White, P., H. Aberle, and J.P. Vincent. 1998. Signaling and adhesion activities of mammalian beta-catenin and plakoglobin in Drosophila. J Cell Biol. 140:183-195.

Winter, C., G., W. Bruce, B. Anna, R. Anne, R. Karess, J. Axelrod, D., and L. Luo. 2001. Drosophila Rho-Associated Kinase (Drok) Links Frizzled-Mediated Planar Cell Polarity Signaling to the Actin Cytoskeleton. Cell. 105:81-91. 


\section{MATERIALS and METHODS}

625

626

627

628

629

630

631

632

633

634

635

636

637

638

639

640

641

642

643

644

645

646

647

648

649

650

651

652

653

654

655

656

657

\section{Fly stocks and genetics.}

Myosin was visualized in live germaria using myosin regulatory light chain ( $s q h$ in Drosophila) fused to GFP, sqh::GFP (Royou et al., 2002) or mCherry, sqh::mCherry (Martin et al., 2008) expressed under its own promotor. Actin was visualized using the F-actin binding domain of Utrophin fused to GFP (Utr::GFP) expressed under an ubiquitous promotor ( $s q h$ promotor, (Rauzi et al., 2010)) or using the UASp-LifeAct::GFP (Huelsmann et al., 2013) construct express in the germline using the nanos-GAL4-VP16 (nos-GAL4, (Van Doren et al., 1998) driver. To visualize ROCK in live, we used a wild-type ROCK allele fused to GFP expressed under an ubiquitous promotor, ubip-GFP::ROCK (Bardet et al., 2013). To visualize DE-Cad in live, we used a knock-in insertion of GFP at the DE-Cad locus (Huang et al., 2009), and to visualize Armadillo we used the stock arm::GFP (BDSC\#8556). Microtubules were visualized in live using the microtubule-associated protein Jupiter fused to GFP, Jup::GFP (Morin et al., 2001). Photoconversion experiments were performed using the stock w; sqh::Dendra2 (Roubinet et al., 2017).

For knockdown experiments, the following stocks were used: v; UASp-white-shRNA (BDSC \#35573), v;; UASp-chic-shRNA (BDSC \#34523), v; UASp-zip-shRNA (BDSC \#37480), v; UASp-mbs-shRNA (BDSC \#41625), v; UASp-shg-shRNA (BDSC \#38207), v; UASp-arm-shRNA (BDSC \#35004). For this study, we generated: sqh::GFP; UASp-chicshRNA, w; UASp-zip-shRNA; Utr::GFP, w; UASp-mbs-shRNA; Utr::GFP using stocks previously described.

Germline clones mutant for $s h g^{R 69}$ or $s h g^{I G 29}$, which are null alleles of $s h g$, rarely induced encapsulation defects in contrast to $s h g$-shRNA. We believed it is caused by the functional compensation between E-Cad and N-Cad in the Drosophila germline as published previously for multinucleation phenotypes (Loyer et al., 2015). As shown by Loyet et al., shg-shRNA does not trigger compensation by N-Cad, we thus used this combination to disrupt cellular adhesion. In addition, we obtained identical encapsulation phenotypes with arm-shRNA, which links both E-Cad and N-Cad to the actomyosin cytoskeleton.

The white-shRNA was used as a control (ctl-RNAi) because white is not expressed during oogenesis. Depending on the strength of the shRNA, different drivers were used for knockdowns in the germline. To knockdown zip and $m b s$ we used nos-GAL4, either the original stock or w; sqh::GFP; nos-GAL4 (generated using stocks previously described). To knockdown chic and shg we used a bam-GAL4(x2) driver (containing two copies of the bam- 
658 GAL4 driver, (Clemot et al., 2018)): w; bam-GAL4(x2) or w; bam-GAL4(x2); Utr::GFP

659 (generated using stocks previously described). For gene knockdowns in follicle cells, we 660 used: w; Traffic jam-GAL4; Utr::GFP (generated using stocks previously described and from

661 Bloomington). Knock downs were performed at $29^{\circ} \mathrm{C}$ to increase the efficiency of the GAL4 662 driver.

663 To generate Flp out clones we used the stock: w hs-Flp; UASp-GFP; act-FRT-y+-FRT-

664 GAL4 (generated using stocks from Bloomington). Heat-shocks were performed on early 665 pupae, $30 \mathrm{~min}$ at $37^{\circ} \mathrm{C}$.

$666 r o k^{2}$ and $s h q^{1}$ clones were generated using the Flp/FRT technique. The following stocks were 667 used: w FRT-19A rok $^{2}$ (Winter et al., 2001), w hs-Flp FRT-19A ubi-mRFP.nls and w FRT ${ }^{101}$ 668 sqh $^{1}$ (Karess et al., 1991), w hs-Flp FRT ${ }^{101}$ ubi-GFP. To induce clones, heat-shocks were 669 performed on L2 larvae for $1 \mathrm{~h}$ at $37^{\circ} \mathrm{C}$ for two consecutive days.

670 To modify cortex properties, favoring or reducing blebs occurrences, we expressed tagged 671 version of Moesin in the germline using nos-GAL4 driver. w; UASp-moe-TA::GFP, w; 672 UASp-moe-TD::GFP are gifts from A. Guichet. We also used: w; UASp-moe-TA::GFP; 673 Utr::GFP and w; UASp-moe-TA::GFP; Utr::GFP (generated using stocks previously described). Crosses were done at $29^{\circ} \mathrm{C}$ to increase the efficiency of the GAL4 driver.

\section{Live and fixed imaging.}

677 5-day-old females were collected and dissected for live imaging or fixed experiments.

678 Live imaging in hydrogel was adapted from (Burnett et al., 2018). Ovaries were dissected in

679 Schneider medium (Sigma-Aldrich), and transfer onto a round $25 \mathrm{~mm}$ coverslip. The coverslips were previously coated with 3-(trimethoxysilyl)propyl methacrylate (SigmaAldrich). Medium was removed and $15 \mu \mathrm{L}$ of 10\% PEG-DA hydrogel solution (esibio) with $0.1 \%$ I2959 (photo initiator, Sigma-Aldrich) was added on the samples. A coverslip treated with deperlent was placed over the hydrogel droplet and the coverslip/coverslip sandwich was then placed over a UV light source and illuminated for 30 s at $312 \mathrm{~nm}$ for gelation. The upper coverslip was removed and the coverslip supporting the hydrogel disc was then placed into a chamber (Chamlide) filled with Schneider medium. See Supplemental Figure 1b. All imaging was performed at $25^{\circ} \mathrm{C}$.

688 Drugs treatment. Few microliters of chemical or vehicle were added directly to the culture 689 chamber while imaging. Cyto-D (Enzo Life Sciences) and CK-666 (Sigma) were diluted in 690 DMSO (Sigma). Final concentrations in Schneider medium after addition of the drugs to the 691 culture chamber were $2.5 \mu \mathrm{M}$ for Cyto-D and 50 $\mu \mathrm{M}$ for CK-666. Y27632 (Enzo Life 
692 Sciences) was diluted in water and added to the chamber for a final concentration of $500 \mu \mathrm{M}$.

693 Colcemid (Sigma) was added for a final concentration of $62 \mu \mathrm{g} \cdot \mathrm{mL}^{-1}$.

694 For live imaging in oil, ovaries were dissected in oil (10S, Voltalef, VWR) and transfer onto a 695 coverslip. Germaria were made to stick to the coverslip in oil.

696 For immunostaining, ovaries were dissected in PBS, fixed in 4\%PFA, permeabilized in PBT

697 (0.2\% Triton) for $30 \mathrm{~min}$, left overnight with primary antibodies in PBT at $4^{\circ} \mathrm{C}$, washed 3

698 times $30 \mathrm{~min}$ in PBT, left with secondary antibody for $2 \mathrm{~h}$ at room temperature, washed 3

699 times $30 \mathrm{~min}$ in PBT and mounted in Cityfluor. The following primary antibodies were used:

700 Orb (Mouse, 1:500, Developmental Studies Hybridoma Bank, DSHB, 4H8) and DE-

701 Cadherin2 (Rat, 1:50, DSMB, Hybridoma Product DCAD2). Secondary antibodies used were

$702 \mathrm{Cy} 3$ and Cy5 (1:200, Jackson laboratories). AlexaFluor568 phalloidin (Invitrogen) was used

703 to visualize F-actin (1:400), and DAPI (Invitrogen) was used at 1:200.

704 All images were acquired on an inverted spinning-disc confocal microscope (Roper/Nikon)

705 operated by Metamorph 7.7 coupled to a sCMOS camera and with a 60X/1.4 oil objective.

706

\section{Image processing and analysis.}

708 Images were processed using Fiji and Imaris (Bitplane) and graphs were generated in Prism

709 (GraphPad). A bleach correction was applied to time-laps images. Images of the movies represent a maximum intensity $\mathrm{Z}$ projection $(15 \mu \mathrm{m})$. Waves and blebs frequency (Figure 1, 2 and 3) were measured in Fiji, every occurrence of a wave or bleb was counted, the resulting number was then divided by the time of the recording. Wave occurrences were either counted

713 per cell (Figure 1f) or per cysts (Figures 1i, 2b, 3b). To measure the average number of blebs

714 per cyst (Figure 5), we counted the number of blebs per cyst for each time frame (every 30 sec) and divided by the number of frames. This method was used to take into account bleb 716 persistence.

717 Region 3 cyst aspect ratios (Figure 3) were measured in Fiji using the build-in toolbox, an

718 ellipse was fitted to the shape of the cyst (as indicated in Figure 3f).

719 Cyst displacement were tracked in Imaris. Cap cells at the anterior tip of the germarium were 720 used as a fixed reference point. To estimate cyst displacement, we tracked the oldest ring 721 canal of each cyst, which is the widest and brightest, making it an easy object to track. We 722 projected displacement along the a-p axis using Imaris build-in toolbox and calculated the mean speed over 1 to $2 \mathrm{~h}$. By convention, we conferred a negative value for displacement speed toward the anterior and a positive value for displacement speed toward the posterior. 
725 To calculate the percentage of stalk width reduction over $50 \mathrm{~min}$ in hydrogel $v s$. in oil, we

726 measure the width of the stalk at $\mathrm{t}=0$ and at $\mathrm{t}=50 \mathrm{~min}$ and divided the difference by the

727 initial width.

728

729 Quantification and statistical analyses.

730 Statistical analyses were performed using the Prism (Graphpad) statistics toolbox. For waves

731 frequency and aspect ratio, $P$ values were calculated using an unpaired $t$-test, the reference

732 sample is the distribution in wild-type. To compare cysts displacements speed in our different

733 conditions, we used a non-parametric Mann-Whitney $U$-test. the reference sample is the

734 distribution in $c t l-R N A i$. Statistical analyses were performed on absolute value of cyst

735 displacement speed. To compare constriction of the stalk in hydrogel vs. oil, $P$ value was

736 calculated using an unpaired $t$-test, the reference sample is the distribution in hydrogel.

737 Cysts autonomous migration on stalled FCs (Figure 6b) was tested using a non-parametric

738 one sample Wilcoxon test. $P$ values were calculated against the null hypothesis $\mathrm{m}=0$ (no

739 displacement). 


\section{FIGURE LEGENDS}

Figure 1: Germ cells generate actomyosin traveling waves associated with pressure

\section{released blebs}

744 (a) Scheme of a germarium. The germarium is divided into four morphological regions along

745 the anterior-posterior axis. At the anterior tip, the germline stem cell and its progeny divide to

746 produce 16-cell germline cysts that are connected by ring canals. The cysts then enter meiosis.

747 At the transition between region $2 \mathrm{a}$ and $2 \mathrm{~b}$ resides follicle stem cells. They produce follicle

748 cells (gray) that surround and separate germline cysts (orange arrows) in a process called

749 encapsulation. In region 3 the cyst adopts the shape of a sphere and is surrounded by one

750 sheet of somatic cells, the newly formed egg chamber will be progressively pinch out of the

751 germarium. Anterior is on the left, posterior on the right.

752 (b) Temporal projection of a 30 min movie of a germarium expressing $s q h:: G F P$ that stains 753 myosin.

754 (c) Time-laps images of $s q h:: G F P$ signal showing myosin travelling waves around the cell

755 cortexes of a germline cyst in region 3. On the last image, waves displacement is represented 756 with time-colored dots.

757 (d) Time-laps images of LifeAct::GFP signal that stains F-actin expressed specifically in the 758 germline showing actin travelling waves around the cell cortexes of a germline cyst in region

759 3. On the last image, waves displacement is represented with time-colored dots.

760 (e) Kymograph showing three consecutive waves of $s q h:: G F P$ (myosin) around the cortex of 761 one cell from a cyst in region $2 b$.

762 (f) Quantification of waves frequency depending on the developmental time of germline cyst

763 cells. $n=6$ GSC or Cystoblast, $n=12$ cells of 2 -cell cysts, $n=21$ cells of 4 -cell cysts, $n=33$

764 cells of 8 -cell cysts, $n=61$ cells of region 2 a cysts, $n=45$ cells of region $2 b$ cysts, $n=42$

765 cells of region 3 cysts; 4 germaria.

766 (g) Time-laps images of a germ cell expressing both rok::GFP (green) and sqh::mCherry

767 (myosin, magenta). Green and magenta arrows point to the position (middle) of rok and

768 myosin waves respectively.

769 (h) Temporal projection of $5 \mathrm{~min}$ movies of germline cysts in region 3 before (left) or after

770 (right) drug or vehicle addition to the medium as indicated. Time is color-coded, such that

771 rainbow signal indicates a travelling wave. Myosin $(s q h: \because G F P)$ or F-actin $($ utr::GFP) waves

772 are examined. 
773 (i) Quantification of waves frequency per cyst in region $2 b$ or 3 before and after drug or

774 vehicle treatments. Mean and standard deviations (SD) are shown. $n=4$ germaria treated with

775 water, $\mathrm{n}=5$ germaria treated with DMSO, $\mathrm{n}=6$ germaria treated with Y27632, $\mathrm{n}=6$

776 germaria treated with Cyto-D, $\mathrm{n}=5$ germaria treated with CK-666.

777 (j, top) cytoplasmic expression of GFP in the germline reveals the presence of bleb

778 protrusions (arrows). (bottom) still-images following a bleb formation and retraction,

779 sqh::GFP stains myosin.

780 (k) Quantification of bleb frequency per cysts in region 2b and region 3. Mean and SD are 781 shown. $\mathrm{n}=19$ region $2 \mathrm{~b}$ cysts, $\mathrm{n}=13$ region 3 cysts, 19 germaria.

782 Scale bars, $10 \mu \mathrm{m}(\mathrm{b}, \mathrm{j}), 5 \mu \mathrm{m}(\mathrm{c}, \mathrm{d}, \mathrm{g}, \mathrm{h})$. See also Movies 1-6 and Supplementary Figure 1.

Figure 2: Alteration of germline contractility induces the formation of abnormal egg chambers

786 (a) Temporal projection of 30 min movies of germaria expressing $s q h:: G F P$ to follow myosin waves in the indicated mutant conditions. Germlines cysts are outlined with yellow dotted 788 lines.

(b) Quantification of wave frequency per cyst in region 2 and 3 of the germarium. Indicated

RNAi were expressed specifically in the germline. Mean and SD are shown. $n=5$ cysts in region $2 \mathrm{a}, \mathrm{n}=8$ cysts in region $2 \mathrm{~b}, \mathrm{n}=8$ cysts in region 3,5 ctl-RNAi germaria; $\mathrm{n}=5$ cysts in region $2 \mathrm{a}, \mathrm{n}=4$ cysts in region $2 \mathrm{~b}, \mathrm{n}=5$ cysts in region 3,5 chic-RNAi germaria; $\mathrm{n}=4$ cysts in region $2 \mathrm{a}, \mathrm{n}=6$ cysts in region $2 \mathrm{~b}, \mathrm{n}=5$ cysts in region 3,3 zip-RNAi germaria; $\mathrm{n}=5$ cysts in region $2 \mathrm{a}, \mathrm{n}=9$ cysts in region $2 \mathrm{~b}, \mathrm{n}=7$ cysts in region 3,5 mbs-RNAi germaria. $* P<$ $0.05, * * P<0.01, * * * P<0.001$, ns, not significant, $t$-test.

(c) Fixed images of ovarioles stained with phalloidin to mark actin (magenta), Orb that marks the oocyte (yellow) and DAPI that marks the DNA (blue). Depletion of zip, chic or mbs in the germline (zip-RNAi, chic-RNAi, mbs-RNAi) lead to the formation of egg chambers with abnormal number of germ cells. White dotted lines underline two different cysts packaged together in one egg chamber (both made of 16 cells). Numbers indicate the number of cells included in each unit. Asterisks mark the position of the two oocytes of the two mbs-RNAi cysts. (Note: $m b s-R N A i$ induces smaller ring canals leading to dedifferentiation of the oocyte and progressive loss of Orb accumulation).

804 (d) Quantification of the occurrence phenotypes (egg chambers made of abnormal of germ cells) in fixed ovarioles for the different mutant conditions. 
(e, left) fixed mosaic ovariole showing a GFP+ zip-RNAi cyst that is split between two egg chambers (numbers indicate the number of cells from the original cyst that have been distributed between the two egg chambers). (right) fixed mosaic ovariole showing one GFP+ $m b s-R N A i$ cyst packaged with an unmarked wild-type cyst within the same egg chamber. Scale bars, $10 \mu \mathrm{m}$ (a), $20 \mu \mathrm{m}$ (c, e). See also Supplementary Figure 2 and Movies 7.

Figure 3: Alteration of germline contractility leads to defective sorting during encapsulation.

814 (a) Still images from movies of $c t l-R N A i$ and zip-RNAi germaria. Germline cysts were tracked 815 over time. Cap cells at the anterior of the germarium serve as a reference point. Original cyst 816 position is indicated with dashed lines and final cyst position is indicated with plain line.

817 White arrows indicate cyst displacement. Red bracket indicates long stalk.

818 (b) Quantification of cyst displacement speed along the a-p axis depending on the different 819 mutant conditions. Violin plots with median and 25\%-75\% quartiles are shown. $\mathrm{n}=44 \mathrm{ctl}$ $820 R N A i$ cysts; $\mathrm{n}=56$ chic- $R N A i$ cysts; $\mathrm{n}=54$ zip-RNAi cysts; $\mathrm{n}=63$ mbs-RNAi cysts. $* * * P<$

8210.001 , ns, non-significant, Mann-Whitney $U$-test (performed on absolute speed values).

822 (c, left) Schematic of a region 3 germline cyst surrounded by somatic cells. To measure the 823 elongation of the cyst along the a-p axis, we measured the aspect ratio of the fitted ellipse 824 (red) as illustrated. (right) Quantification of region 3 cysts aspect ratio depending on the 825 different mutant conditions. Dot and whisker plots are shown. $\mathrm{n}=26$ ctl-RNAi, $\mathrm{n}=30$ chic$826 R N A i, \mathrm{n}=25$ zip-RNAi, $\mathrm{n}=38 m b s-R N A i . * P<0.05$, ns, non-significant, $t$-test. See also Supplementary Figure 3 and Movies 8-9

\section{Figure 4: Cadherin-based adhesion is required for correct encapsulation}

830 (a) Wild type germarium stained with E-Cad (magenta) and F-actin (green). E-Cad localized 831 within germline cells around ring canals (plain arrows) and between germline cysts and the 832 somatic layer (empty arrows).

833 (b) Quantification of wave frequency per cyst in region 2 and 3 of the germarium. Mean and 834 SD are shown. $\mathrm{n}=6$ cysts in region $2 \mathrm{a}, \mathrm{n}=8$ cysts in region $2 \mathrm{~b}, \mathrm{n}=6$ cysts in region 3,5 835 ECad-RNAi germaria. ns, not significant, $t$-test.

836 (c) Temporal projection of a 30 min movie of a ECad-RNAi germarium expressing sqh::GFP 837 to follow myosin waves.

838 (d) Fixed images of ovarioles stained with phalloidin to mark actin (magenta), Orb that marks 839 the oocyte (yellow) and DAPI that marks the DNA (blue). Depletion of ECad in the germline 
840 (ECad-RNAi germinal) or in the follicle cells (Ecad-RNAi somatic) lead to encapsulation

841 defects and the formation of egg chambers with abnormal number of cerm cells. White dotted

842 lines underline two different cysts packaged together in one egg chamber. Numbers indicate

843 the number of cells included in each unit.

844 (e) Quantification of the occurrence of encapsulation phenotypes in fixed ovariols when Ecad 845 is depleted in the germline (Ecad-RNAi germ.) or in the somatic cells (Ecad-RNAi soma.).

846 (f) Quantification of region 3 cysts aspect ratio depending on the different mutant conditions.

847 Dot and whisker plots are shown. $\mathrm{n}=26$ ctl-RNAi, $\mathrm{n}=33$ ECad-RNAi germ, $\mathrm{n}=17$ ECad-

848 RNAi soma. ** $P<0.01$, ns, not significant, $t$-test.

849 (g) Quantification of cyst displacement speed along the a-p axis depending on the different

850 mutant conditions. Violin plots with median and 25\%-75\% quartiles are shown. $\mathrm{n}=53 \mathrm{ECad}$ -

851 RNAi germ cysts; $\mathrm{n}=39$ ECad-RNAi soma cysts. *** $P<0.001$, Mann-Whitney $U$-test

852 (performed on absolute speed values).

853 (h) Still images from movies of Ecad-RNAi germ and ECad-RNAi soma germaria. Germline

854 cysts were tracked over time. Cap cells at the anterior of the germarium serve as a non-

855 moving reference point. Original cyst position is indicated with dashed lines whereas final

856 cyst position is indicated with plain line. White arrows indicate cyst displacement

857 Anterior is on the left and posterior on the right. Scale bars, $10 \mu \mathrm{m}(\mathrm{c}), 20 \mu \mathrm{m}(\mathrm{a}, \mathrm{d})$. See also

858 Movies 10-13 and Supplementary Figure 4.

860 Figure 5: Increasing blebs frequency induces forward movements of germline cysts and 861 cyst collisions.

862 (a) wild-type germarium (left) and germaria overexpressing moe-TA::GFP or moe-TD::GFP

863 in the germline (middle and left) stained with Orb (yellow) and phalloidin (actin, magenta).

864 Cysts boundaries are underline in white. Bleb protrusions are indicated with arrows.

865 (b) Quantification of the average number of blebs per cyst in region 2b and 3, depending on

866 the different conditions. Mean and SD are shown. $n=7$ cysts in region $2 b, n=9$ cysts in

867 region 3, 12 wild type germaria; $n=12$ cysts in region $2 b, n=27$ cysts in region 3, 20 moe-

$868 T A: \because G F P$ germaria, $\mathrm{n}=8$ cysts in region $2 \mathrm{~b}, \mathrm{n}=11$ cysts in region 3,10 moe-TD::GFP

869 germaria. $* P<0.05$, ns, non-significant, $t$-test.

870 (c) Fixed images of ovarioles stained with phalloidin to mark actin (magenta), Orb that marks

871 the oocyte (yellow) and DAPI that marks the DNA (blue). (left) Overexpression of moe-

$872 T A:: G F P$ in the germline lead to cyst collisions. The invading anterior cysts are underline in 
873

874

875

876

877

878

879

880

881

882

883

884

885

886

887

888

889

890

891

892

893

894

895

896

897

898

899

900

901

902

903

904

905

906

white. (Right) Compound egg chamber made of a moe-TA::GFP overexpressing cyst and one unmarked wild-type cyst.

(d) Quantification of the occurrence of encapsulation phenotypes in fixed ovarioles with germinal overexpression of moe-TA::GFP or moe-TD::GFP.

(e) Quantification of cyst displacement speed along the a-p axis depending on the different conditions. Only cysts in region 3 that have already transition from disc-shape to a rounder shape were considered. Violin plots with median and $25 \%-75 \%$ quartiles are shown. $\mathrm{n}=21$ cysts in region 3 for moe-TA::GFP; $\mathrm{n}=12$ cysts in region 3 for moe-TD::GFP. ns, nonsignificant. $* * P<0.01$, Mann-Whitney $U$-test (performed on absolute speed values).

(f) Still images from a movie of a germarium overexpressing moe-TA::GFP in the germline. An anterior cyst (yellow outline) migrate forward and collide with a posterior cyst.

Scale bars, $20 \mu \mathrm{m}$. See also Movies 14-15.

\section{Figure 6: Germline cyst can migrate when somatic cell movement is blocked.}

(a, left) Schematic of the posterior part of the germarium. FCs form a stalk that separate the two cysts. Red arrow indicates the measure of the stalk width. (right) Quantification of the percentage of stalk width reduction during 50 min of recording, for germaria mounted either in hydrogel or in oil (negative values indicate expansion). Box and whisker plots are shown. $\mathrm{n}$ $=20$ germaria mounted in hydrogel, $\mathrm{n}=13$ germaria mounted in oil. $* * * P<0.001$, $t$-test.

(b) Quantification of cyst displacement speed along the a-p axis when germaria are mounted in oil. Violin plots with median and 25\%-75\% quartiles are shown. $\mathrm{n}=26$ cyst in Region 3 ctlRNAi; 25 cysts in region 3 for zip-RNAi, 22 cysts in region 3 for shg-RNAi. *** $P<0.001$, ns, non-significant. One sample Wilcoxon signed rank test (test against the null hypothesis $\mathrm{m}$ $=0$, no displacement).

(c) Still images from a movie of a control germarium (ctl-RNAi) mounted in oil. An anterior cyst in region 3 migrate forward and collides with a posterior cyst. Original cyst position is indicated with dashed lines whereas final cyst position is indicated with plain line. White arrows indicate cyst displacement.

(d) Still images of a mosaic germarium containing wild-type (RFP+, magenta) germline cyst and $r o k^{2}$ mutant cysts. (bottom) High magnification are shown. A wild-type cyst (RFP+, red outline) migrates forward and invade the position of a mutant cyst (unmarked, white outline). Red arrow indicates cyst displacement.

See also Movies 16-17 and Supplementary Figure 5. 
Figure 7: Encapsulation requires a proper balance between germline and somatic forces.

908

909

910

911

(left) Germline cysts exert mechanical forces dependent on cortical contractility and adhesion (red arrows). This confers stiffness within the 16-cell cyst to maintain cyst integrity and propelling forces to maintain proper cyst positioning during encapsulation. At the same time, somatic cells migrate around germline cyst exerting constriction forces (orange arrows). In wild type, germline forces resist constriction forces exerted by surrounding somatic cells and maintain correct cyst position during encapsulation. (middle) Modifying the equilibrium between germline and somatic forces decouples cysts movement from somatic cells movement. On one hand, decreasing germline contractility or adhesion induce germline cysts sliding backward or forward, leading to cysts collision, which can lead to the formation of compound egg chambers. decreasing germline contractility or adhesion also induce cyst splitting by constricting somatic cells, which generate incomplete egg chambers. On another hand, increasing germline contractility or blebbing or blocking somatic cells movement can induces a net forward migration of the germline cysts that can also result in cyst collision and encapsulation defects.

\section{Supplementary Figure 1. Related to Figure 1}

(a) photoconversion experiments reveled traveling waves. Time-laps images of a germ cell expressing $s q h:: D e n d r a 2$ that stains myosin. sqh::Dendra2 molecules were photoconverted with blue light $(405 \mathrm{~nm})$ at $\mathrm{t}=0$ within the indicated ROI (dashed line). Bottom panels show converted molecules (revealed with $561 \mathrm{~nm}$ laser).

(b) Schematic of the hydrogel set-up for live imaging. See Methods.

\section{Supplementary Figure 2. Related to Figure 2}

(a) Quantification of wave frequency per cyst in region $2 b$ and 3 of the germarium of wild type and rok ${ }^{2}$ clones. Mean and SD are shown. $\mathrm{n}=10 \mathrm{wt}$ cysts in region $2 \mathrm{~b}, \mathrm{n}=6$ wt cysts in region $3 ; \mathrm{n}=6 r^{r o k^{2}}$ cysts in region $2 \mathrm{~b}, \mathrm{n}=5 r^{r o k^{2}}$ cysts in region $3 ; 14$ mosaic germaria. $* P<$ $0.05, * * * P<0.001, t$-test.

(b) Fixed image stained with Orb that marks oocytes (magenta) and DAPI that marks the DNA (blue) of a compound egg chamber containing a wild type (GFP+) and a $s q h^{1}$ mutant cyst. White dotted line underlines the two cysts packaged together.

\section{Supplementary Figure 3. Related to Figure 3.}


940 Quantification of cyst displacement speed along the a-p axis for the different indicated mutant

941 conditions. The figure represents the same data set as in Figure $3 \mathrm{~b}$ with the detailed

942 displacement speed for cysts either in region $2 \mathrm{~b}$ (positioned roughly in the middle of the

943 germarium and with a disc shape) or in region 3 (positioned at the posterior of the germarium

944 and with a rounder shape). Violin plots with median and 25\%-75\% quartiles are shown. $\mathrm{n}=$

94520 cysts in region $2 \mathrm{~b}, 24$ cysts in region 3 for $c t l-R N A i ; \mathrm{n}=25$ cysts in region $2 \mathrm{~b}, 31$ cysts in

946 region 3 for chic-RNAi; $\mathrm{n}=21$ cysts in region $2 \mathrm{~b}, 33$ cysts in region 3 for $z i p-R N A i ; \mathrm{n}=21$

947 cysts in region $2 \mathrm{~b}, 42$ cysts in region 3 for $m b s-R N A i$. $* P<0.05$, ** $P<0.01$, *** $P<0.001$,

948 ns, non-significant, Mann-Whitney $U$-test (performed on absolute speed values).

\section{Supplementary Figure 4. Related to Figure 4.}

951 (a) Fixed germaria expressing Utr::GFP (actin, green) and stained for E-Cad. (right) E-Cad 952 localized within germline cells around ring canals (plain arrows) and between germline cysts 953 and the somatic layer in wild type (empty arrows). (middle) germline knock down of ECad 954 specifically reduce E-Cad staining between germ cells. (left) somatic knock down of ECad 955 specifically reduce E-Cad staining between somatic cells.

956 (b) Quantification of cyst displacement speed along the a-p axis depending on the different 957 mutant conditions. The figure represents the same data set as in Figure $4 \mathrm{f}$ with the detailed 958 displacement speed for cysts either in region $2 b$ (positioned roughly in the middle of the 959 germarium and with a disc shape) or in region 3 (positioned at the posterior of the germarium 960 and with a rounder shape). Violin plots with median and $25 \%-75 \%$ quartiles are shown. $\mathrm{n}=$ 96125 cysts in region $2 \mathrm{~b}, 28$ cysts in region 3 for shg-RNAi germ.; $\mathrm{n}=17$ cysts in region $2 \mathrm{~b}, 22$ cysts in region 3 for shg-RNAi soma. *** $P<0.001$, Mann-Whitney $U$-test (performed on absolute speed values).

964 (c) Fixed images of ovarioles stained with phalloidin to mark actin (magenta), Orb that marks 965 the oocyte (yellow) and DAPI that marks the DNA (blue). Germline knock down of arm leads 966 to encapsulation defects similar to germline knock down of $s h g$, such as cyst collision within 967 the germarium and cyst splitting.

968 Scale bars, $20 \mu \mathrm{m}$

\section{Supplementary Figure 5. Related to Figure 6.}

971 Comparison of cyst displacement speed along the a-p axis when samples are mounted in 972 hydrogel or in oil for the different conditions as indicated. Violin plots with median and 25\%$97375 \%$ quartiles are shown. For measurements in hydrogel, $\mathrm{n}=44 \mathrm{ctl}$-RNAi cysts; $\mathrm{n}=54$ zip- 
$R N A i$ cysts; $\mathrm{n}=53$ ECad-RNAi cysts. For measurements in oil, $\mathrm{n}=44$ ctl-RNAi cysts; $\mathrm{n}=43$ zip-RNAi cysts; $\mathrm{n}=46$ ECad-RNAi cysts. ${ }^{*} P<0.05$, *** $P<0.001$, Mann-Whitnay $U$-test.

\section{MOVIES LEGENDS}

Movie 1: Cortical myosin waves in germ cells. Related to Figure 1.

Time-lapse images from a germarium expressing the myosin marker sqh::GFP.

Movie 2: Cortical F-actin waves in germ cells. Related to Figure 1.

Time-lapse images from a germarium expressing the F-actin marker LifeAct::GFP in the germline (using the nos-GAL4 driver). The contour of the germarium is outlined.

\section{Movie 3: Blocking myosin activity or actin polymerization using chemical inhibitors} stops wave's propagation. Related to Figure 1.

Time-lapse images from germaria expressing the myosin marker sqh::GFP or the F-actin marker Utr::GFP as indicated. Drugs or vehicle (DMSO) were added to the culture medium at $6 \mathrm{~min}$ as indicated.

\section{Movie 4: Depolymerizing microtubule does not affect wave's propagation. Related to}

\section{Figure 1.}

Time-lapse images from germaria expressing the microtubule marker Jup::GFP (top) or the myosin marker sqh::GFP (bottom) as indicated. Colcemid was added to the culture medium at $6 \mathrm{~min}$ as indicated. Addition of Colcemid immediately depolymerize microtubules but does not affect myosin waves.

\section{Movie 5: Germline cysts are blebbing. Related to Figure 1.}

Time-lapse images from a germarium expressing the myosin marker sqh::GFP and cytoplasmic GFP in the germline (using the nos-GAL4 driver). Arrows indicate blebs protrusion.

Movie 6: Inhibiting cortical contractility by adding Cyto-D immediately stops blebs. Related to Figure 1. 
1007

1008

1009

1010

1011

1012

1013

1014

1015

1016

1017

1018

1019

1020

1021

1022

1023

1024

1025

1026

1027

1028

1029

1030

1031

1032

1033

1034

1035

1036

1037

1038

1039

1040

Time-lapse images from a germarium expressing the myosin marker sqh::GFP and cytoplasmic GFP in the germline (using the nos-GAL4 driver). Cyto-D was added to the culture medium at $15 \mathrm{~min}$.

Movie 7: Genetic modifications of cortical contraction waves. Related to Figure 2.

Time-lapse images from germaria expressing the myosin marker sqh::GFP and the indicated RNAi in the germline. chic-RNAi and zip-RNAi reduce the number of waves per cyst, whereas $m b s-R N A i$ increases the number of waves per cyst.

\section{Movie 8: Reducing germline contractility affects cyst displacement during} encapsulation. Related to Figure 3.

Time-lapse images from germaria expressing the F-actin marker Utr::GFP and the indicated RNAi in the germline. Cysts displacements along the a-p (y) axis were tracked over time. A reference point was position at the anterior end of the germaria were cap cells reside. The displacement track is time-colored from blue to red.

Movie 9: Modifying germline contractility can lead to cyst splitting or collision. Related to Figure 3.

Time-lapse images from germaria expressing the F-actin marker Utr::GFP and the indicated RNAi in the germline. (top) Example of a split cyst in chic-RNAi. (bottom) Example of a collision in $m b s-R N A i$.

Movie 10: Dynamic junctional complexes between germ cells and between germ cells and somatic cells. Related to Figure 4.

Time-lapse images from germaria expressing the junctional markers ECad::GFP (top) or arm::GFP (bottom).

Movie 11: Reducing adhesion affects cyst displacement during encapsulation. Related to Figure 4.

Time-lapse images from germaria expressing the F-actin marker Utr::GFP and shg-RNAi either in the germline (top) or in the somatic cells (bottom). Cysts displacements along the a-p (y) axis were tracked over time. A reference point was position at the anterior end of the germaria were cap cells reside. The displacement track is time-colored from blue to red. 
1041 Movie 12: Decreasing germ cells adhesion can lead to cyst splitting. Related to Figure 4.

1042 Time-lapse images from germaria expressing the F-actin marker Utr::GFP and the indicated

1043 RNAi in the germline. Two examples of cysts being split (arrows) are shown, in $s h g-R N A i$

1044 (top) and in arm-RNAi (bottom).

Movie 13: Decreasing DE-Cad in the germline does not affect cortical waves. Related to

1047 Figure 4.

1048 Time-lapse images from germaria expressing the myosin marker sqh::GFP and the indicated 1049 RNAi in the germline.

1050

1051 Movie 14: Increasing blebs frequency can induce forward cyst movement and cysts

1052 collision. Related to Figure 5.

1053 (top) Time-lapse images from a germarium expressing moe-TA::GFP in the germline (using 1054 the nos-GAL4 driver) and the F-actin marker Utr::GFP. Cyst displacement along the a-p (y) 1055 axis were tracked over time. A reference point was position at the anterior end of the 1056 germarium were cap cells reside. The displacement track is time-colored from blue to red. 1057 (bottom) Example of a collision. Time-lapse images from a germarium expressing moe-

1058 TA::GFP in the germline (using the nos-GAL4 driver). An anterior cyst migrates forward and 1059 invade the position of a more posterior cyst.

Movie 15: Increasing blebs frequency can induce cysts collision. Related to Figure 5.

1062 Time-lapse images from a germarium expressing moe-TA::GFP in the germline (using the nos-GAL4 driver). A higly blebbing cyst invade the position of a more posterior cyst. This germarium was mounted in oil.

Movie 16: Mechanically blocking somatic cells movements can induce forward cysts migration. Related to Figure 6.

1068 Time-lapse images from a germarium expressing the F-actin marker Utr::GFP and the a ctl$1069 R N A i$ in the germline. Cysts displacements along the a-p (y) axis were tracked over time. A 1070 reference point was position at the anterior end of the germarium were cap cells reside. The 1071 displacement track is time-colored from blue to red. The germarium was mounted in oil that 1072 blocks somatic cell centripetal migration. 
1075 Time-lapse images from a mosaic germarium containing wild-type (RFP+) germline cysts and

$1076 r^{2} k^{2}$ mutant cysts (marked by the absence of RFP), and expressing the myosin marker

1077 sqh::GFP. A wild-type cyst (asterisk) migrate and invade the position of a more posterior

1078 mutant cyst. This germarium was mounted in oil.

1079

1080 
a

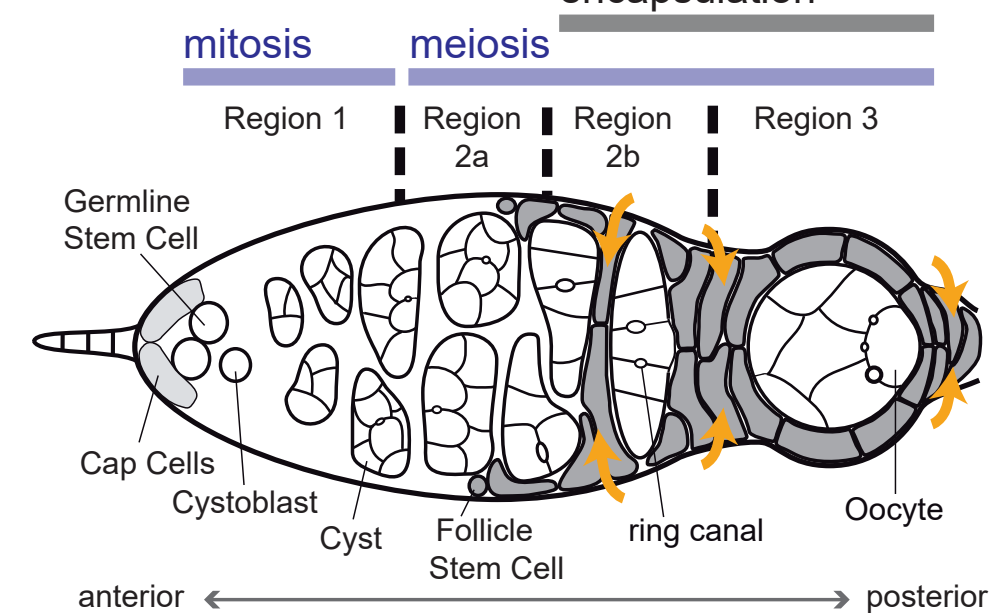

e

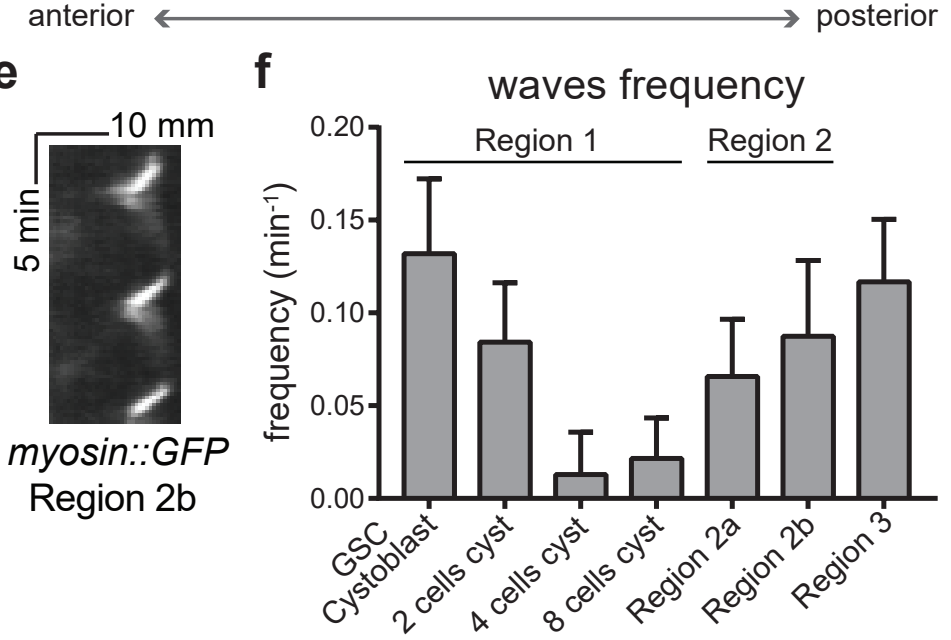

h
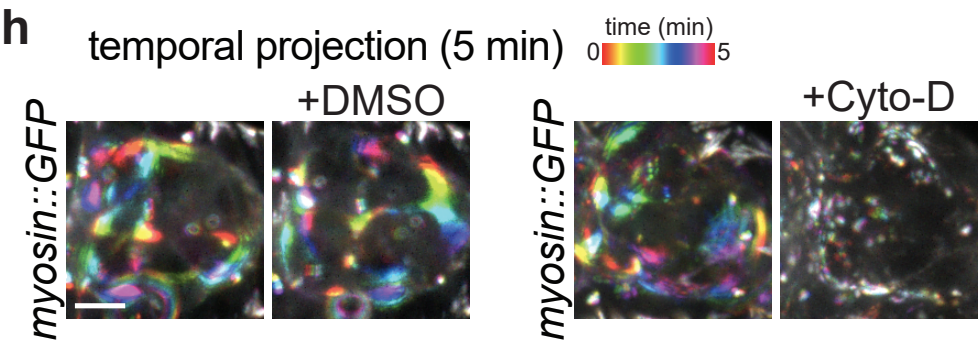$$
\text { i }
$$$$
\text { 足 }
$$

$+Y-27632$
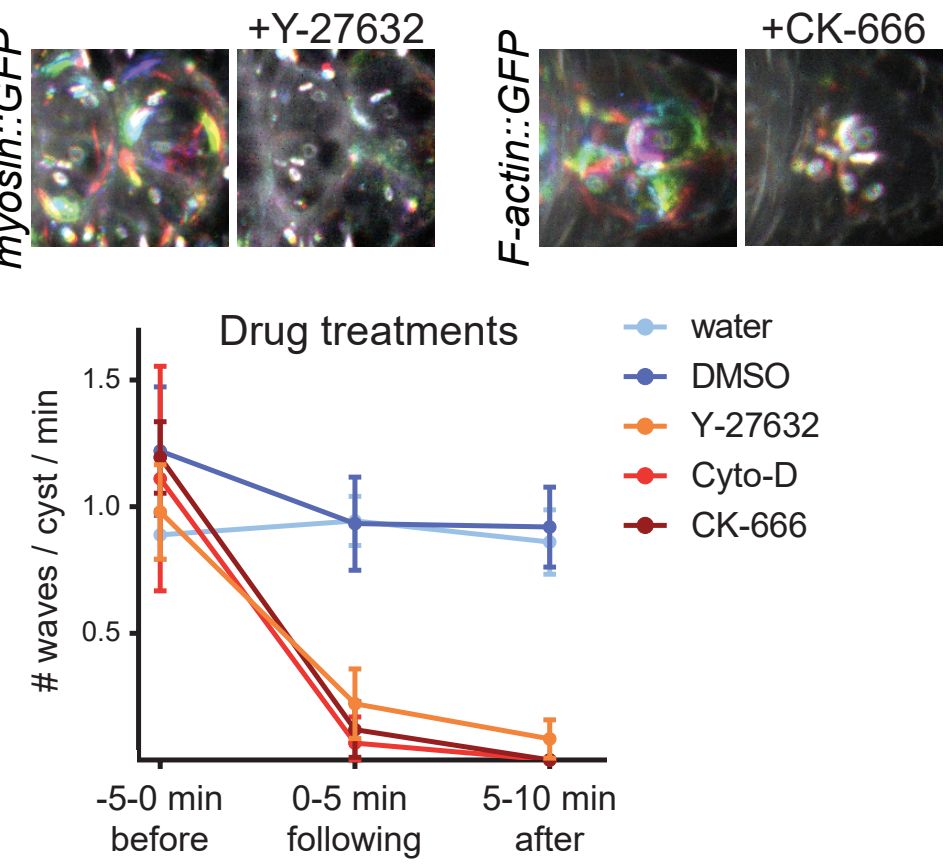

$\mathrm{t}=0 \mathrm{~s}$

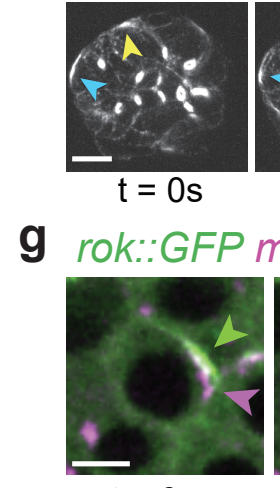

myosin::GFP temporal projection (30 min)

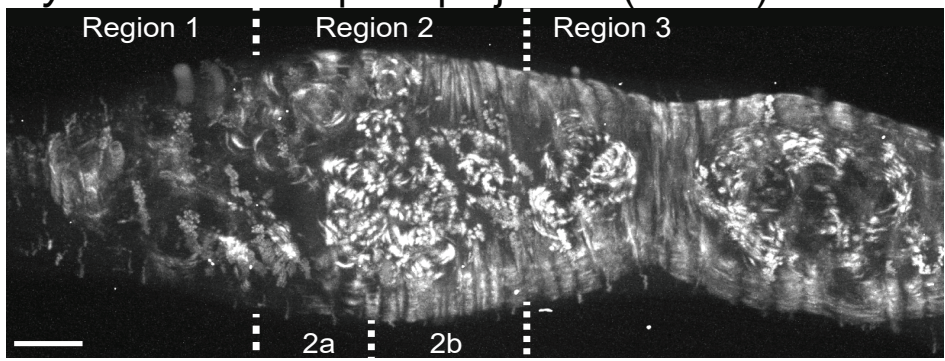

c myosin::GFP (Region 3)

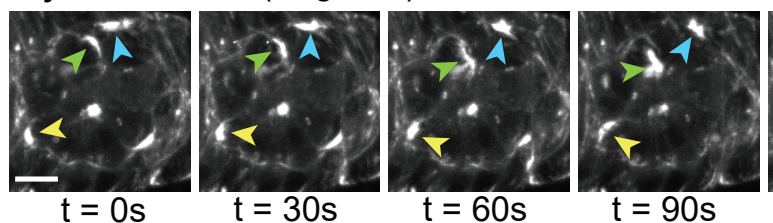

d nos > F-actin::GFP (Region 3$)$ j nos > GFP; myosin::GFP

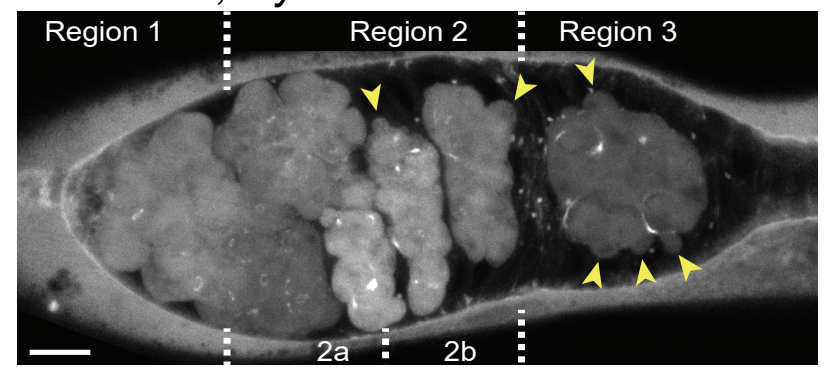

myosin::GFP
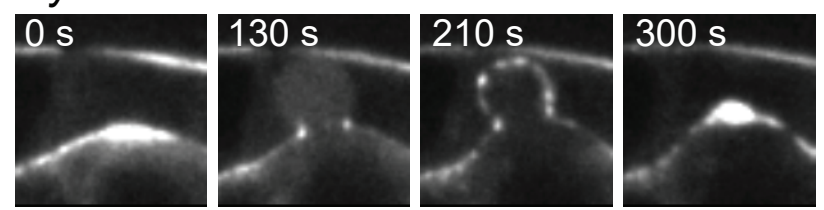

k

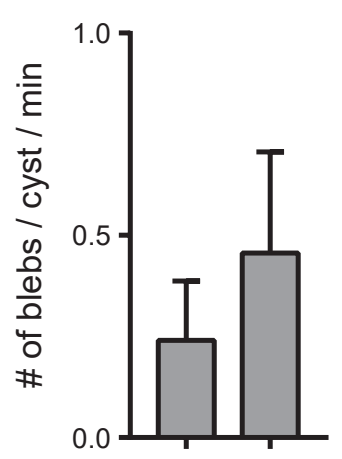

Regions: $2 \mathrm{~b} \quad 3$ 
a myosin::GFP temporal projection (30 $\mathrm{min}) \quad \mathbf{b}$

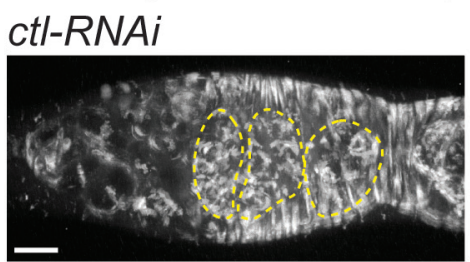

chic-RNAi (profilin)

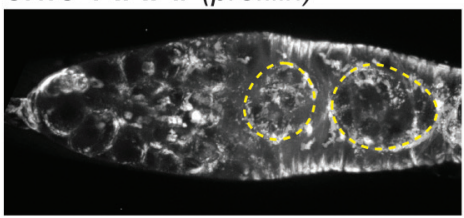
zip-RNAi (myosin)

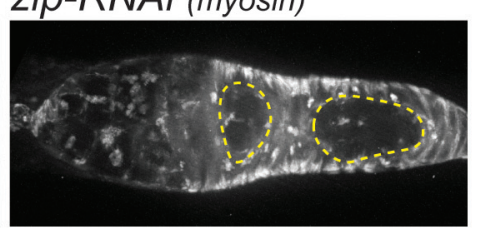

mbs-RNAi (myo. phosphatase)

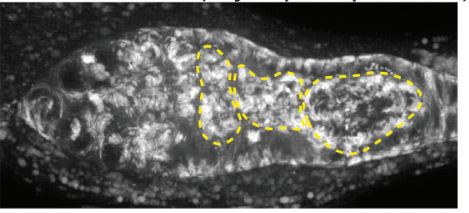

waves frequency

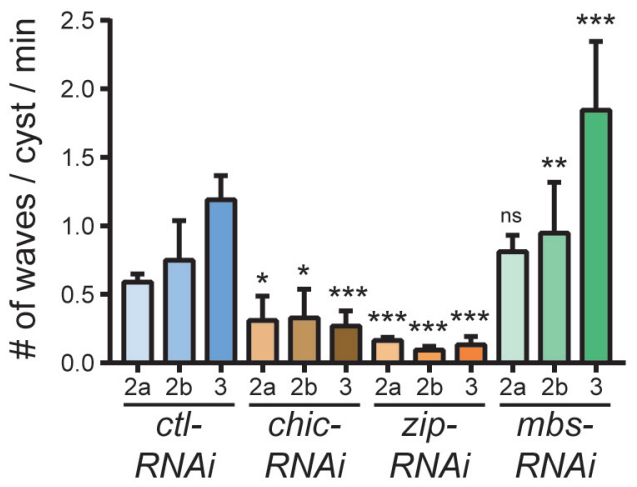

C

ctl-RNAi

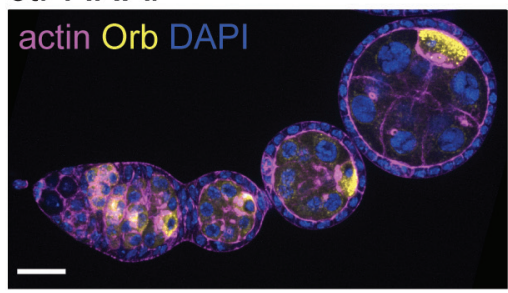

chic-RNAi

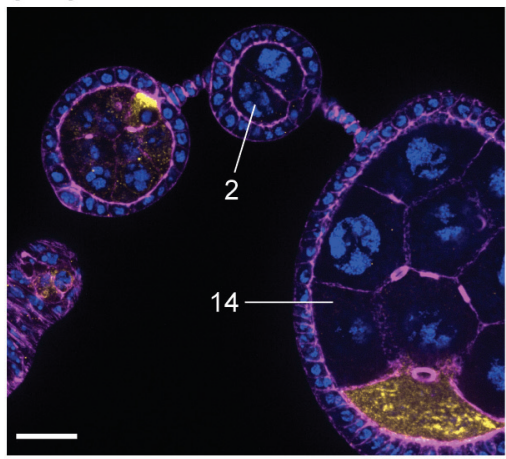

zip-RNAi

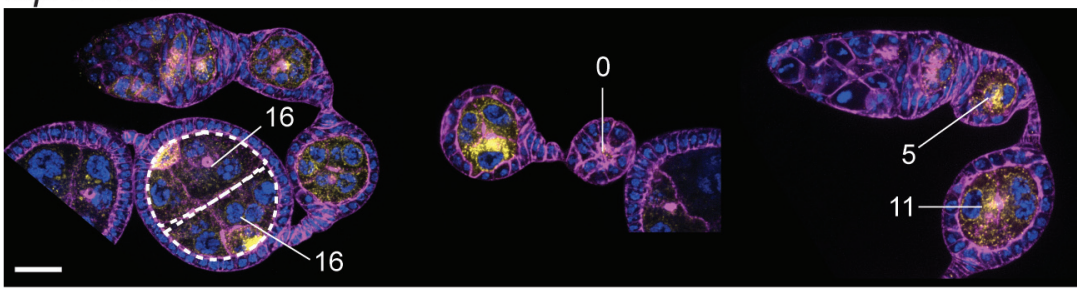

mbs-RNAi

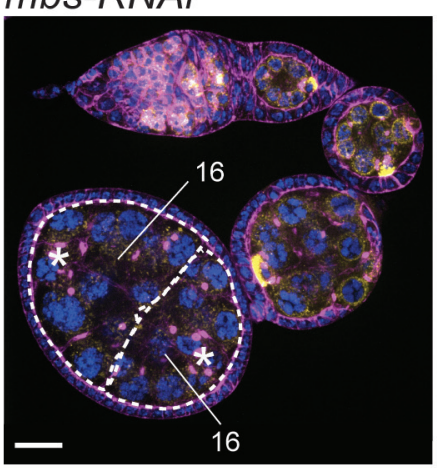

d

$\%$ of ovarioles containing deficient egg-chambers (with abnormal \# of germ cells)

\begin{tabular}{r|c} 
ctl-RNAi $(n=51)$ & 0 \\
chic-RNAi $(n=80)$ & $21 \%$ \\
zip-RNAi $(n=54)$ & $24 \%$ \\
mbs-RNAi $(n=67)$ & $15 \%$
\end{tabular}

e

zip-RNAi clones

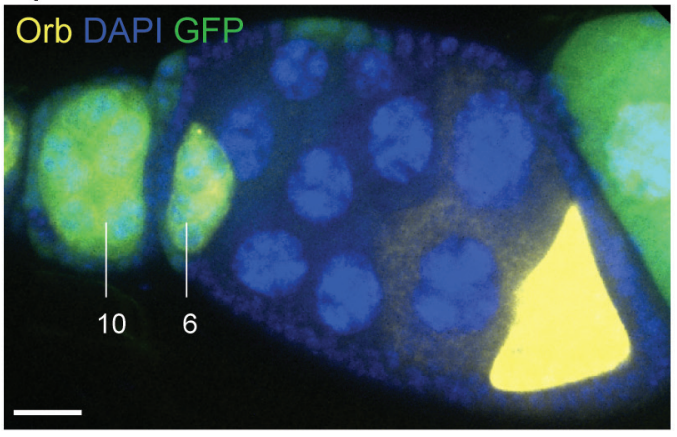

mbs-RNAi clones

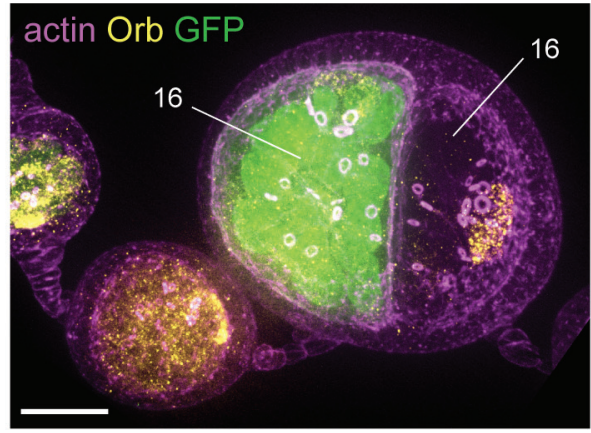

Figure 2 
a

$c t /-R N A i$
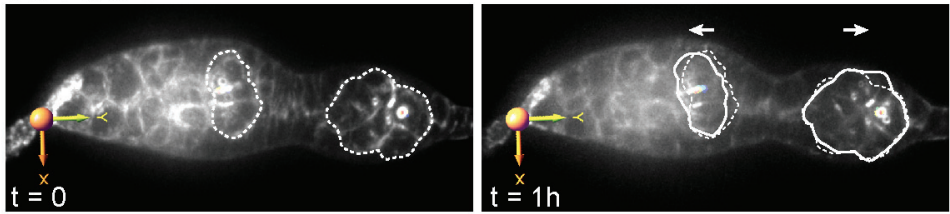

$z i p-R N A i$
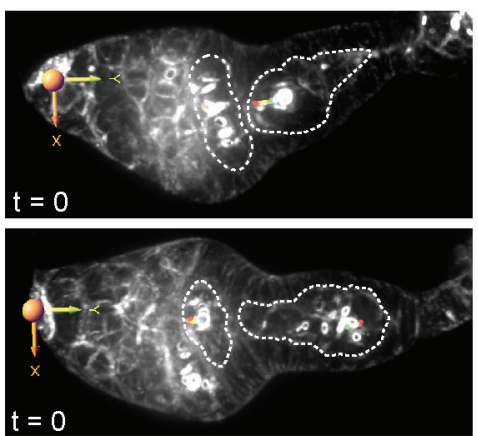

C

Region 3

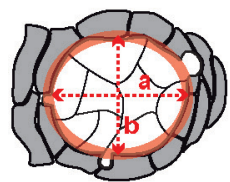

aspect ratio

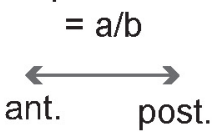

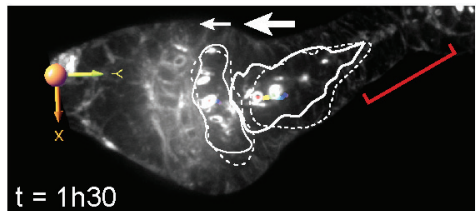

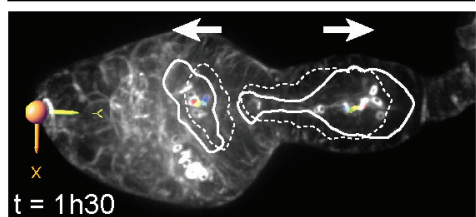

Reg. 3 cysts

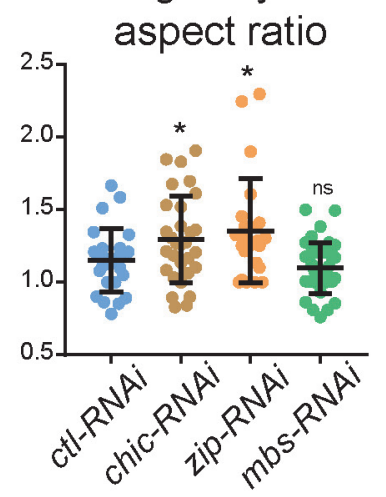

Figure 3

cyst displacement speed $(\mu \mathrm{m} / \mathrm{min})$

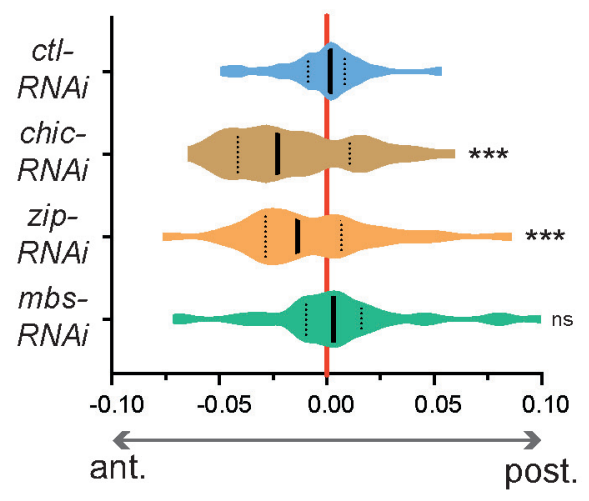




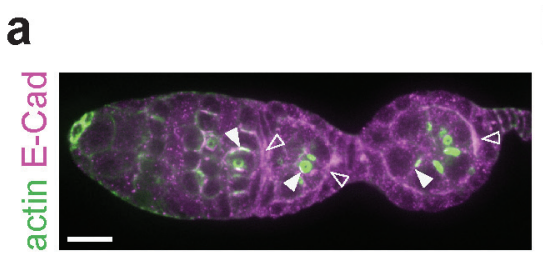

b waves c
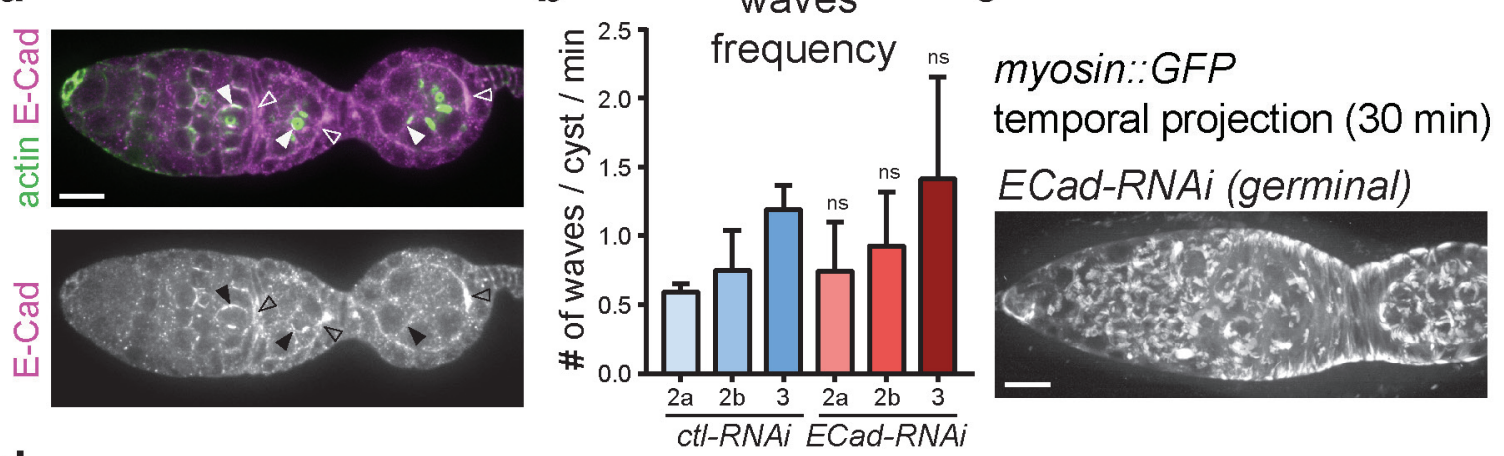

d

ECad-RNAi (germinal)
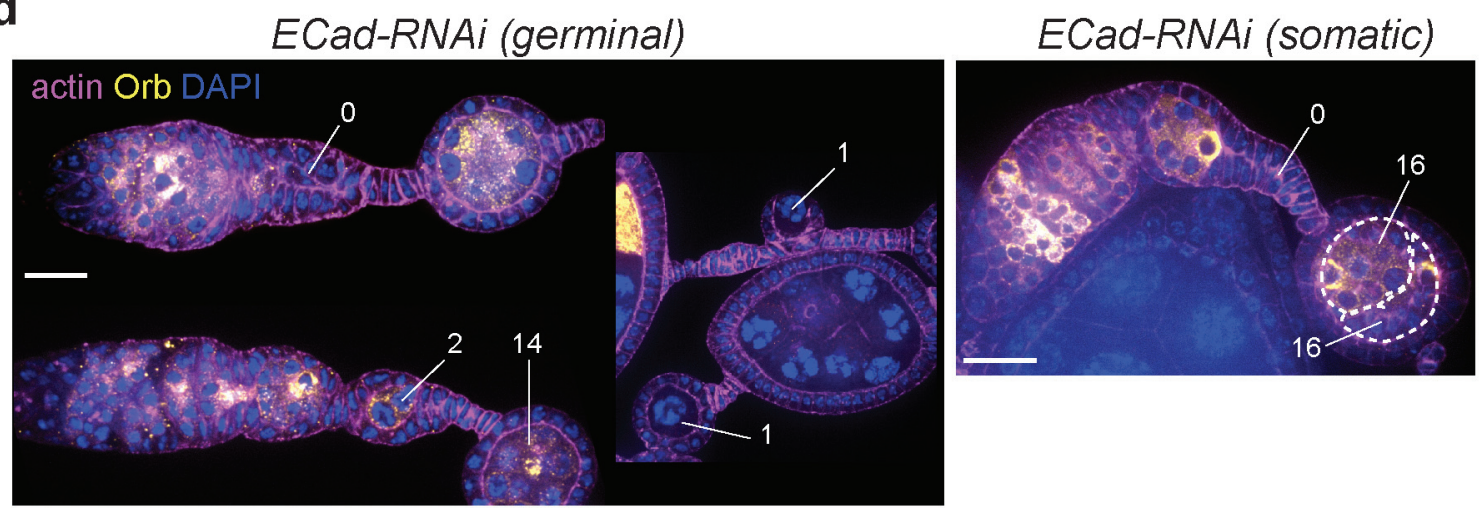

e

$\%$ of ovarioles with

\begin{tabular}{l|l} 
ECad-RNAi germ $(n=70)$ & $71 \%$ \\
ECad-RNAi soma $(n=46)$ & $29 \%$
\end{tabular}

f

Reg. 3 cysts

aspect ratio

g

cyst displacement speed

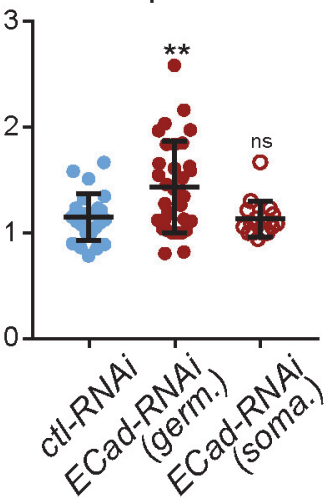

h encapsulation defects $(\mu \mathrm{m} / \mathrm{min})$

ECad-RNAi (germ.)
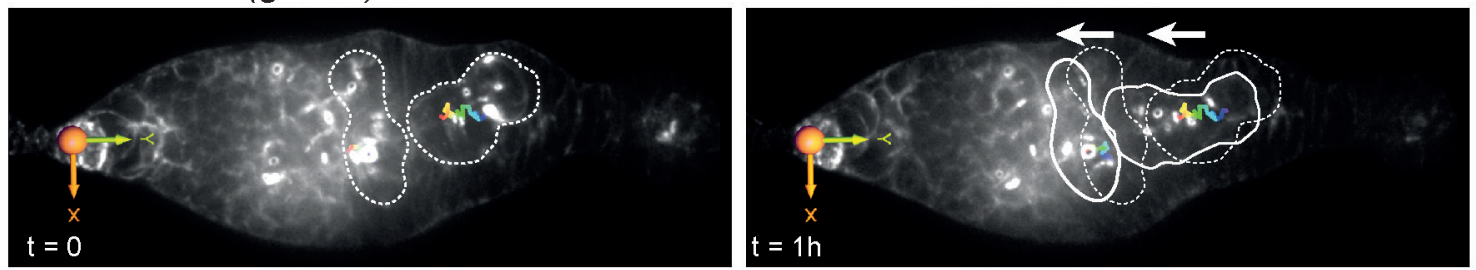

ECad-RNAi (soma.)
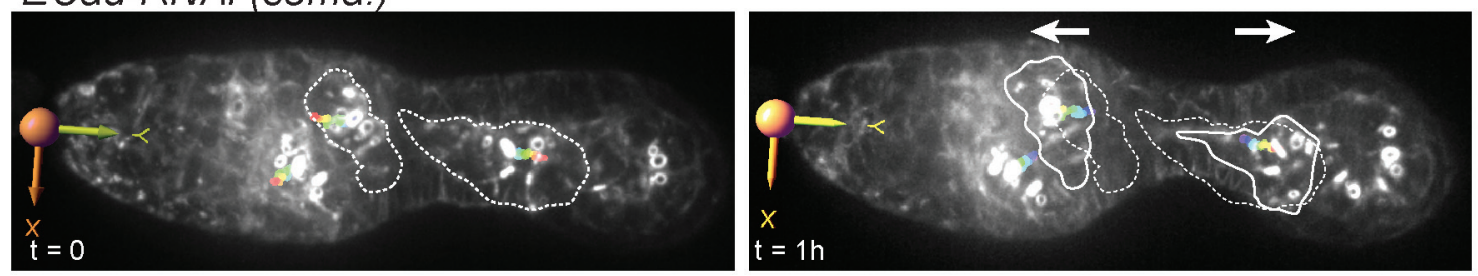

Figure 4 
a

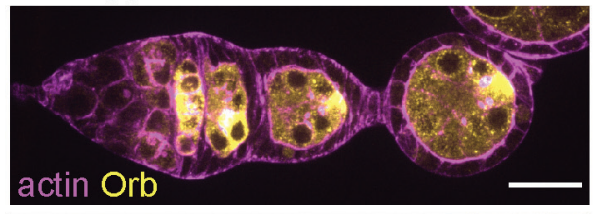

nos $>$ moeTA::GFP
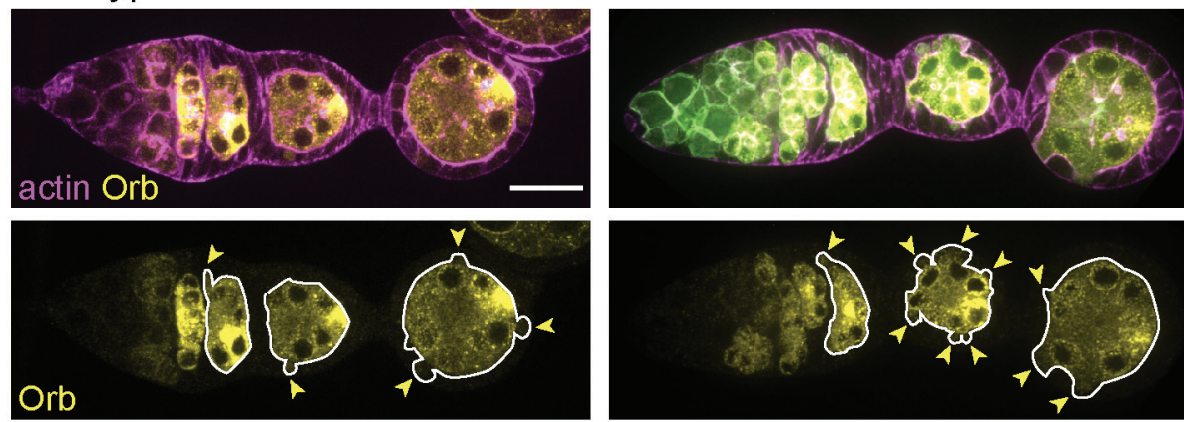

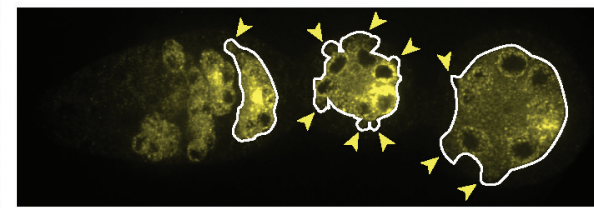

nos $>$ moeTD ::GFP
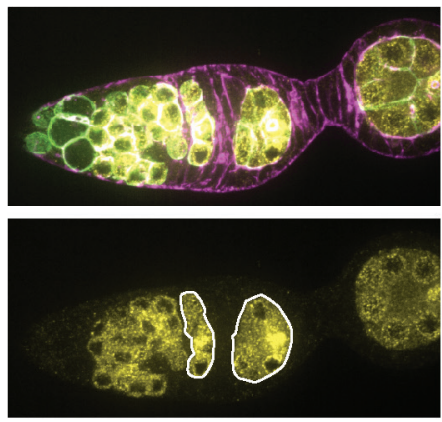

moeTA::GFP clones

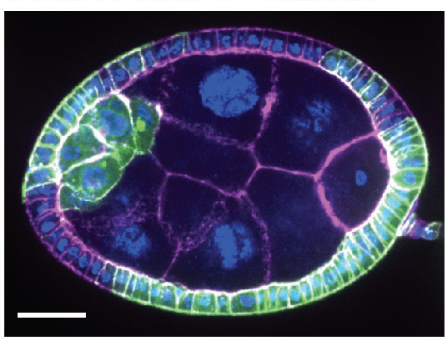

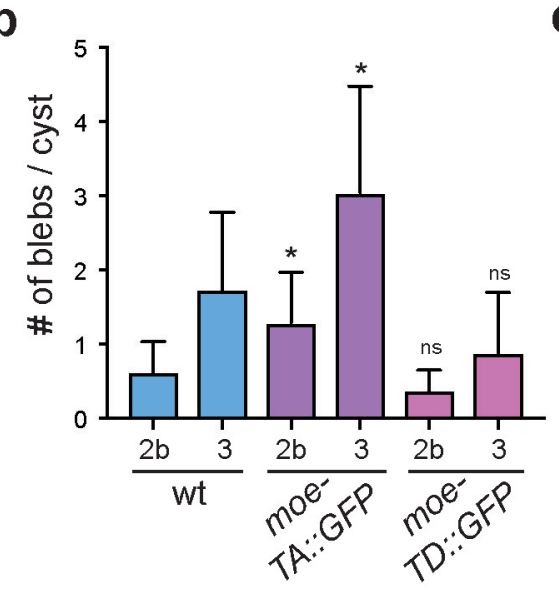

C nos > moeTA::GFP

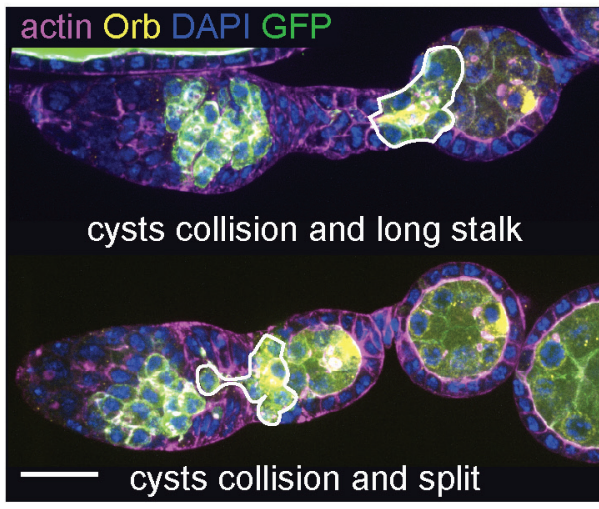

d

$\%$ of ovarioles with encapsulation defects

f nos $>$ moeTA::GFP
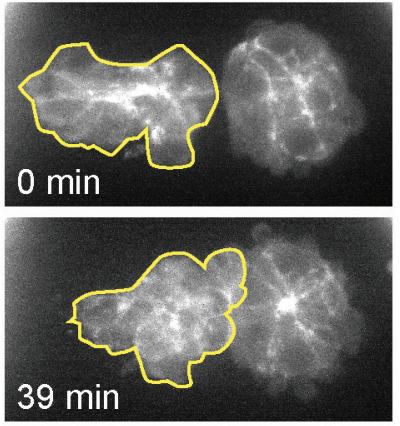

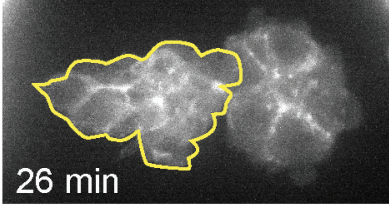

$52 \mathrm{~min}$

moe-TA::GFP $(n=87) \mid 9 \%$ moe-TD::GFP $(n=60) \quad 5 \%$

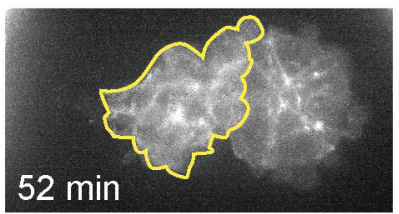

e $\quad$ Reg. 3 cyst displacement speed $(\mu \mathrm{m} / \mathrm{min})$

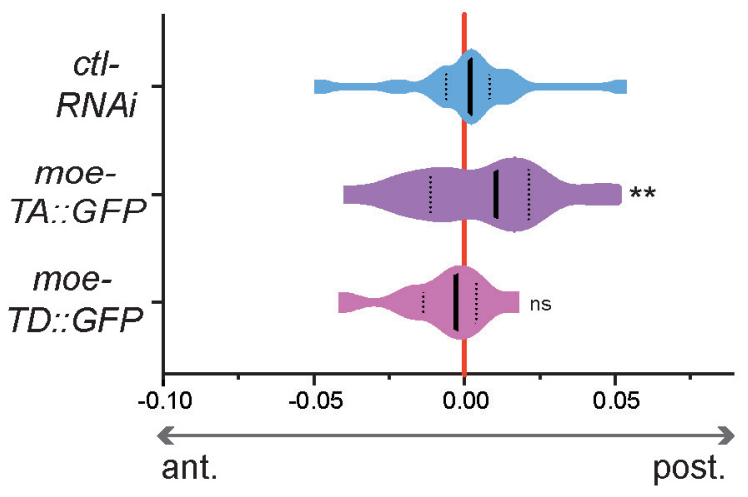

\section{Figure 5}


a

$\%$ of stalk width reduction (in $50 \mathrm{~min}$ )

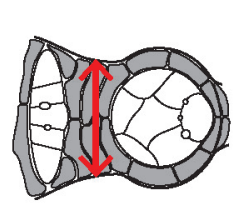

stalk width

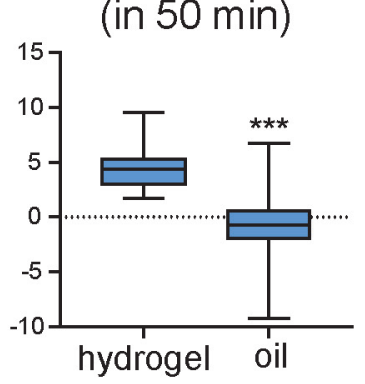

C ctl-RNAi (oil)
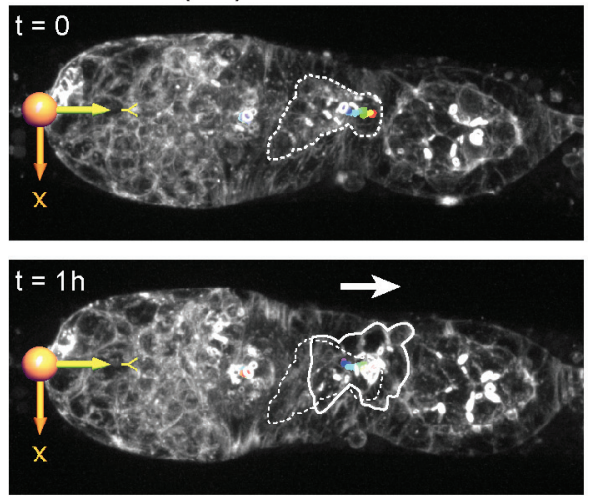

b

Reg. 3 cyst displacement speed d rok ${ }^{2}$ clones (oil) (oil) $(\mu \mathrm{m} / \mathrm{min})$
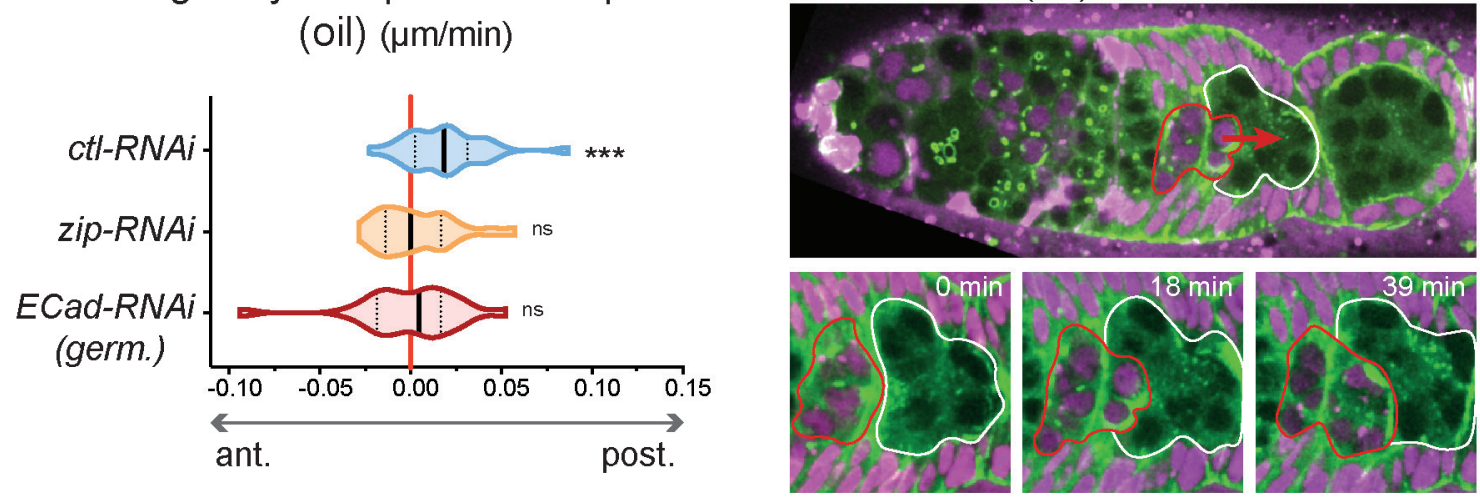

Figure 6 


\section{Decreasing contractility or}

adhesion in the germline

Stiffness

Propelling Forces

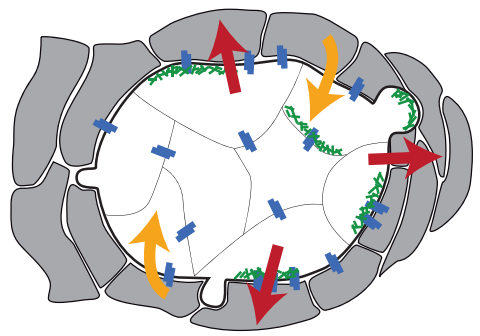

Constriction Forces

actomyosin waves

- E-Cad adhesion complex

Figure 7
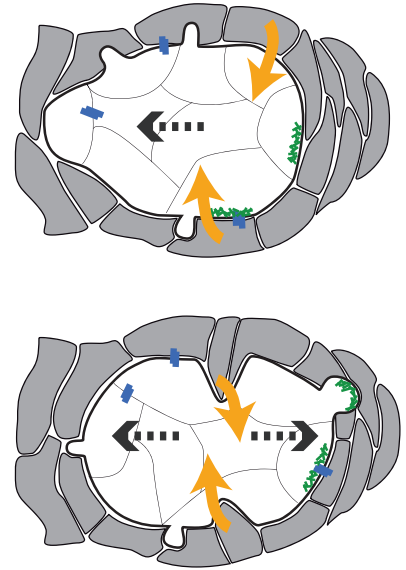

compound egg-chamber

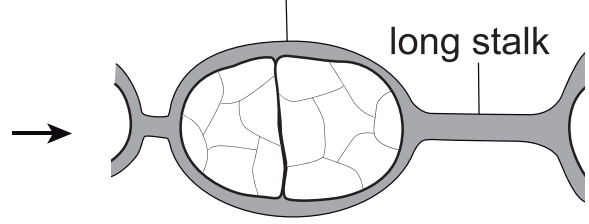

egg-chambers made of split cyst

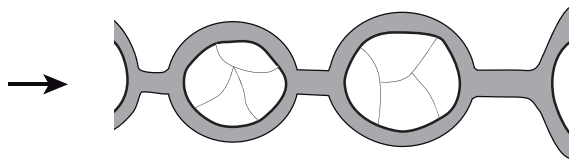

Increasing contractility or blebs in the germline

\section{Blocking somatic cells} movements

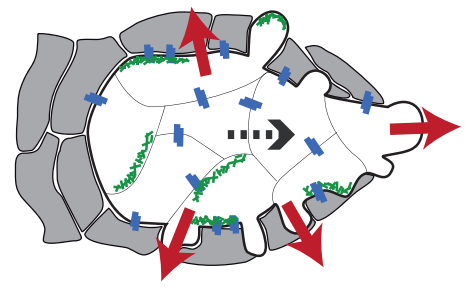

compound egg-chamber

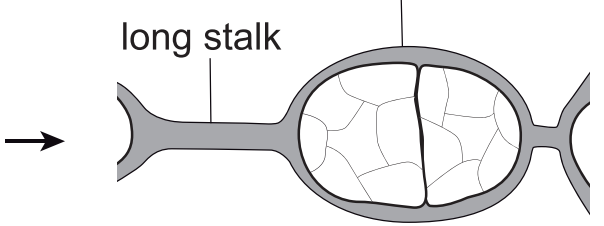


a

sqh::Dendra
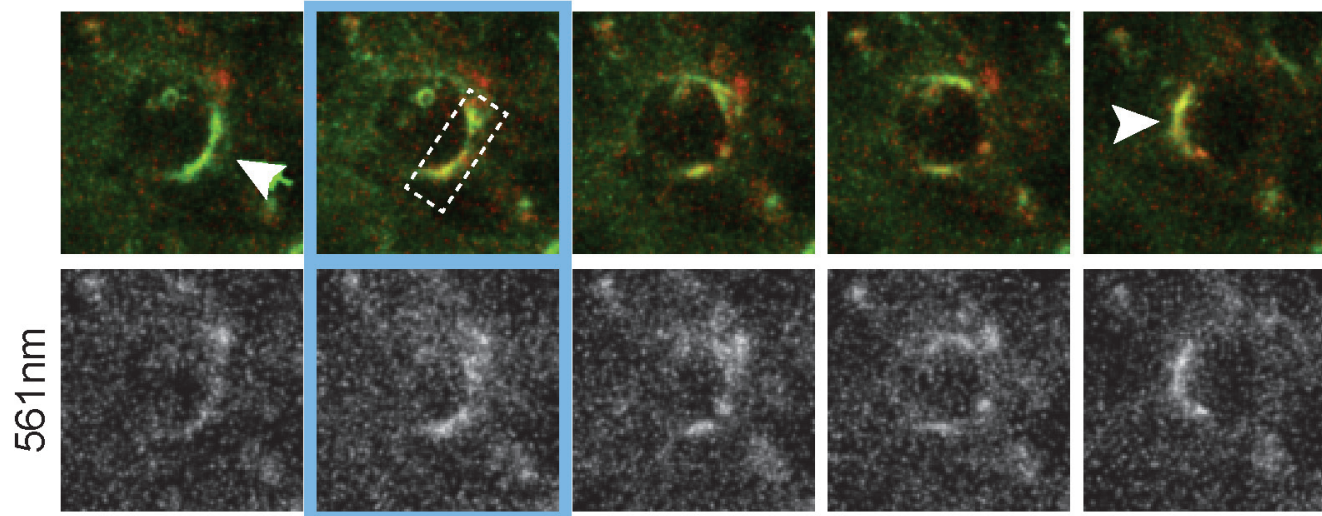

$$
\begin{array}{cc}
t=-10 s \quad t=0 s \\
\text { photoconversion }
\end{array}
$$$$
t=+20 s
$$$$
\mathrm{t}=+40 \mathrm{~s}
$$

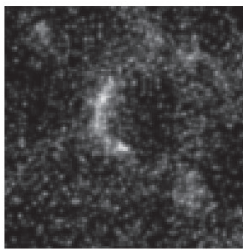

$t=+80 s$

b

Hydrogel set-up for live imaging

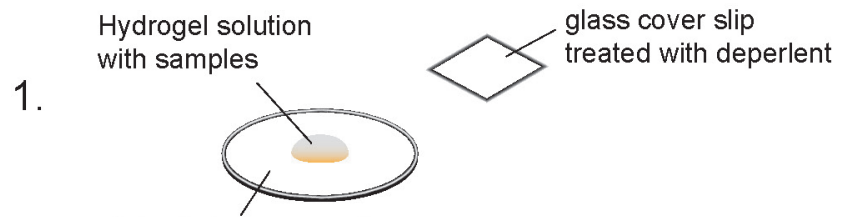

salinized glass cover slip

2.

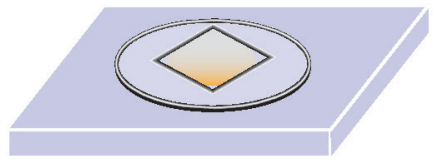

UV lamp
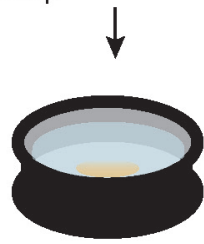

chamber

filled with medium

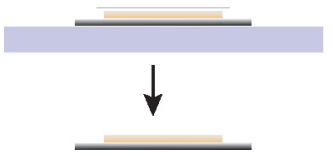

1

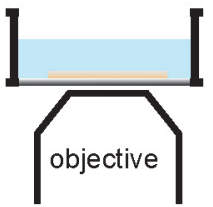




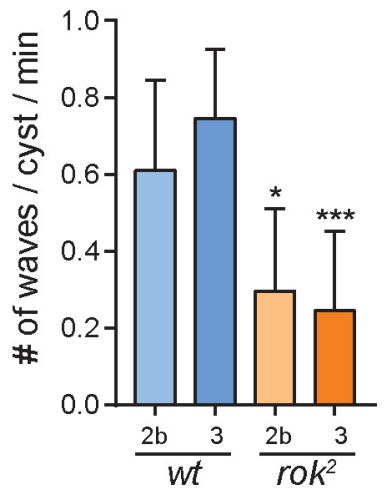
GFP Orb DAPI

clones clones

\section{Figure S2}




\section{cyst displacement speed $(\mu \mathrm{m} / \mathrm{min})$}

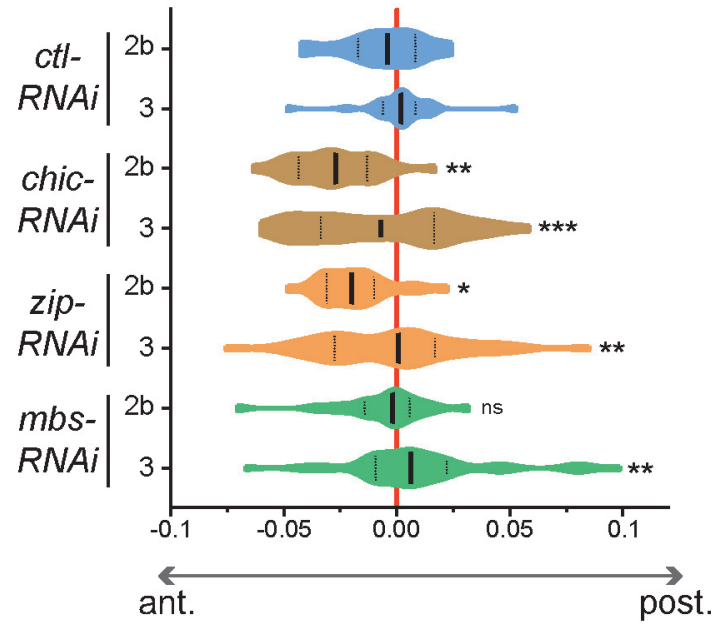

\section{Figure S3}


